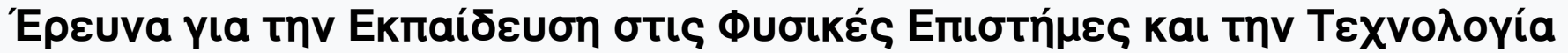

Tóp. 1, Ap. 1 (2021)

\section{Eıઠıเкó TعúXoc}

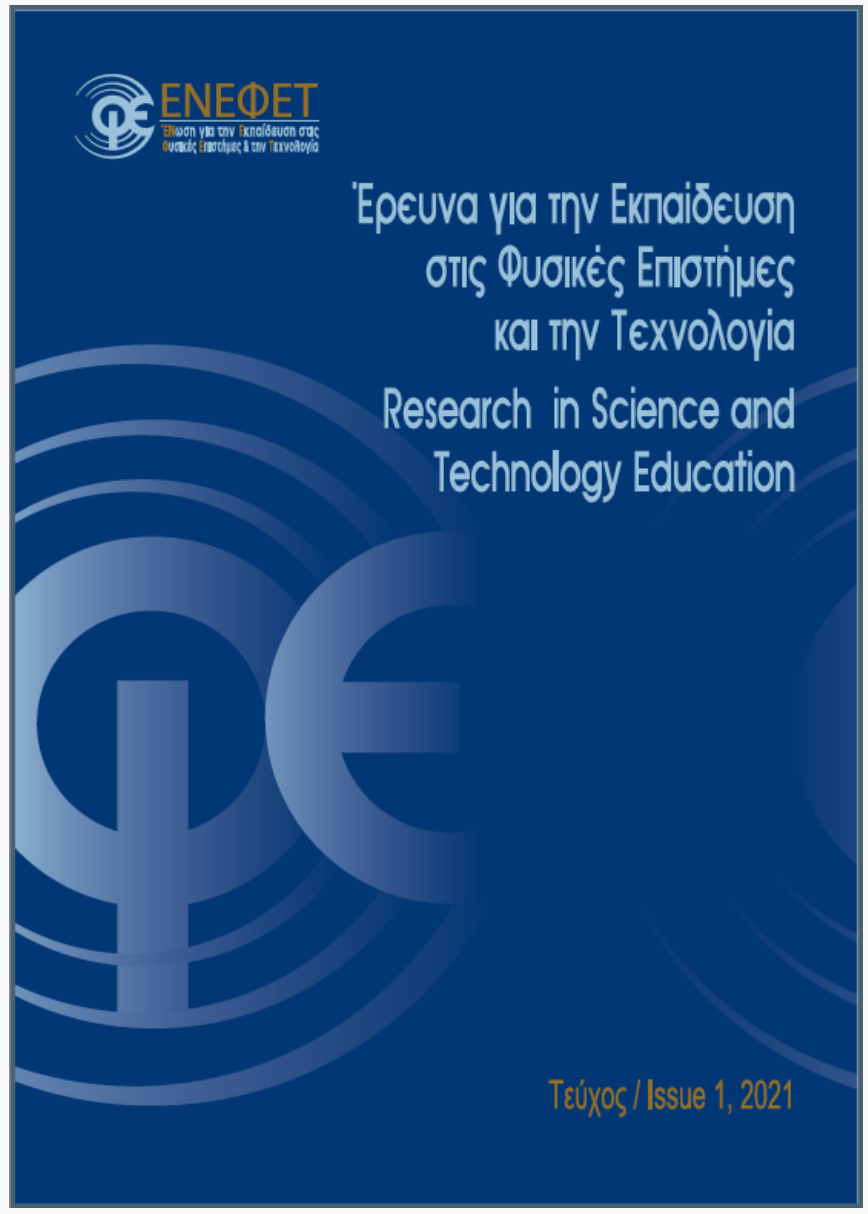

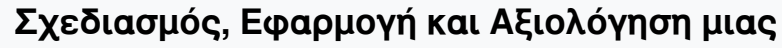

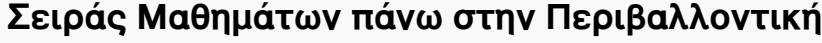

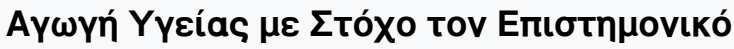

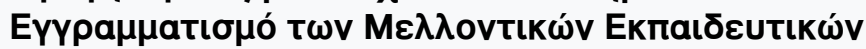

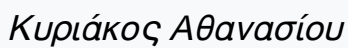

doi: $\underline{10.12681 / \text { riste. } 27266}$

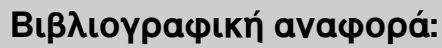

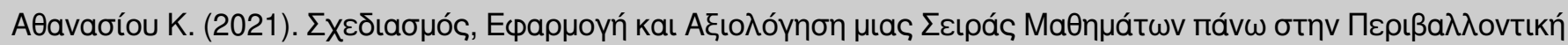

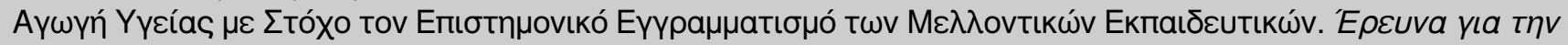

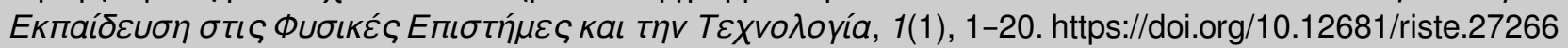




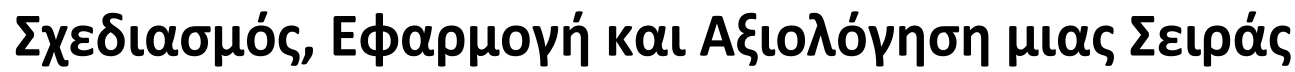

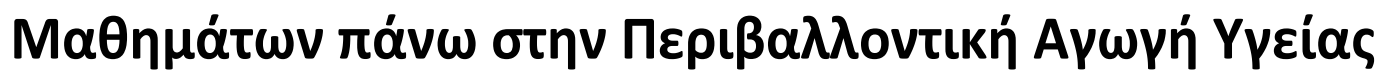

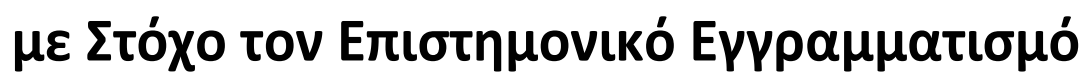

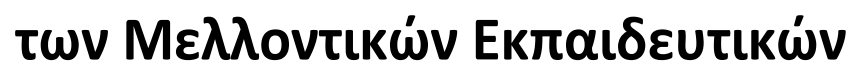

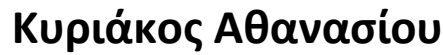

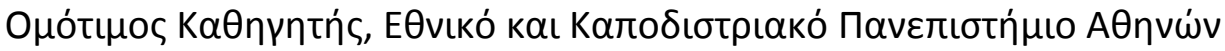 \\ kathanas@ecd.uoa.gr
}

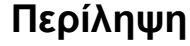

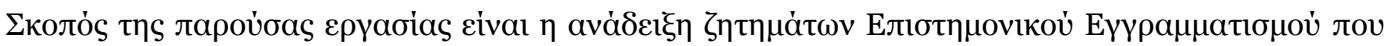

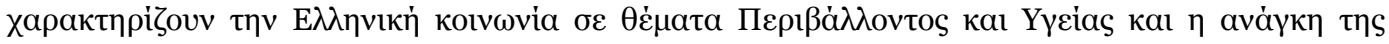

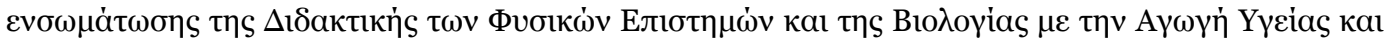

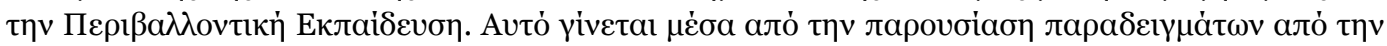

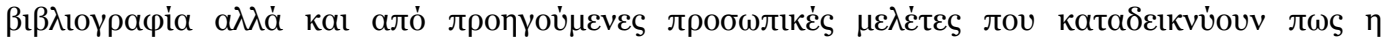

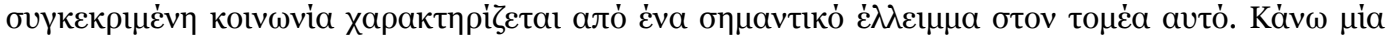

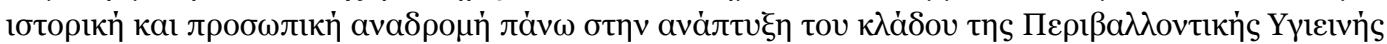

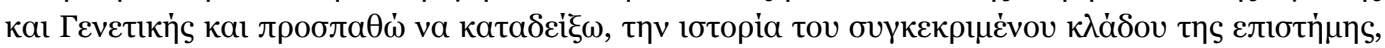

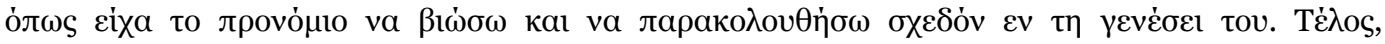

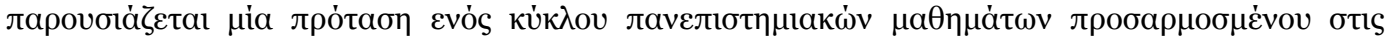

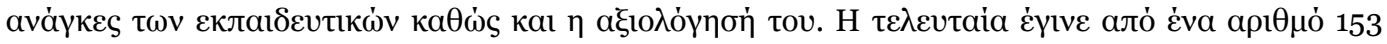

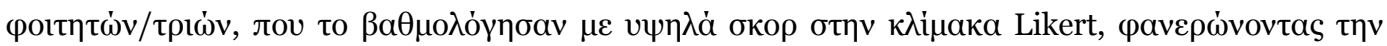

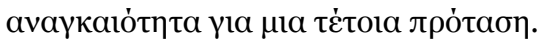

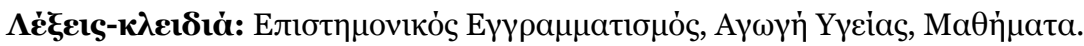

\begin{abstract}
The aim of this paper is to highlight issues of scientific literacy that characterize the Greek society in environmental and health issues, and the need to integrate the Teaching of Science and Biology with Health and Environmental Education. This is accomplished by the means of a presentation of examples from the literature and from previous personal studies, as well, a fact indicating that this
\end{abstract}


society is characterized by a significant deficit in these matters. Then, I take a historical and personal look on the evolution of the field of environmental health and genetics and try to review its history, as I had the privilege of experiencing almost in its birth. Finally, it is presented, a proposal for a university course adapted to the needs of teachers, together with its evaluation. The latter was made through the response to a questionnaire of 153 students, who highly rated the course on the Likert scale, revealing the need for such a proposal.

Key words: Scientific literacy, Environmental health, course.

\section{Eıoaywyń}

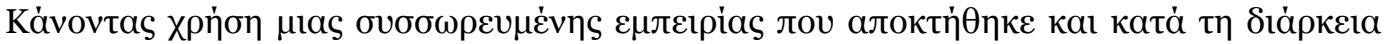

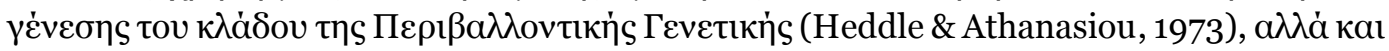

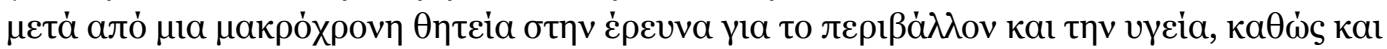

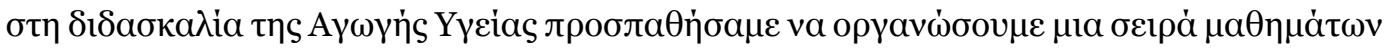

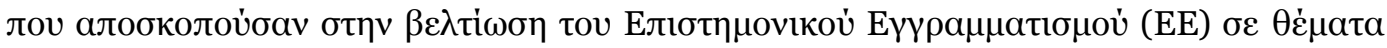

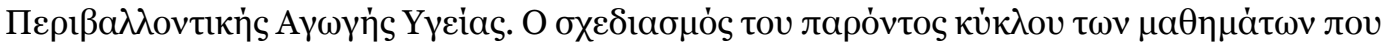

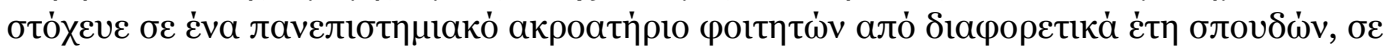

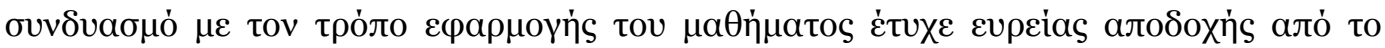

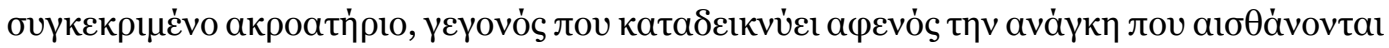

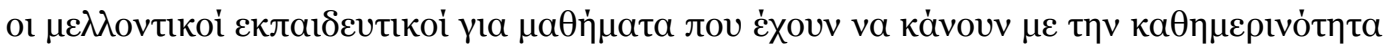

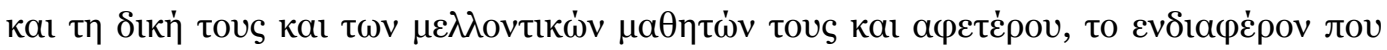

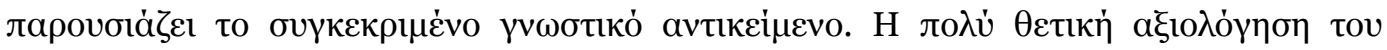

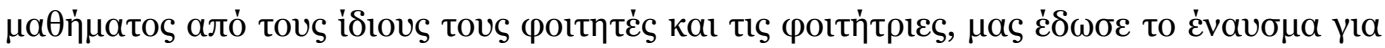

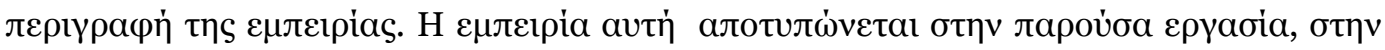

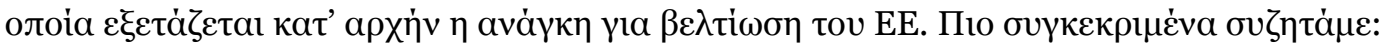

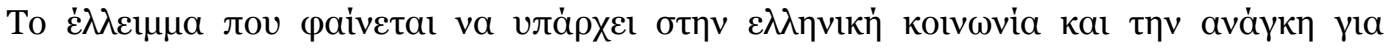

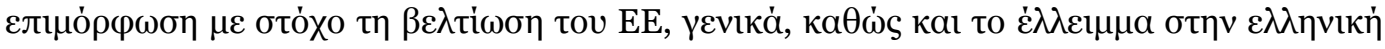

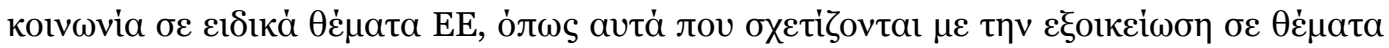

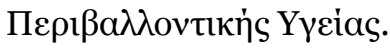

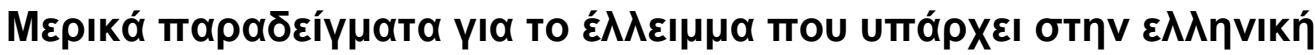

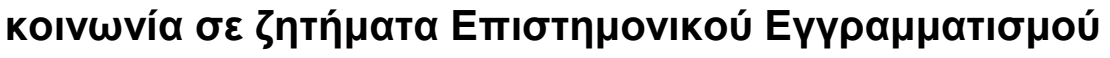

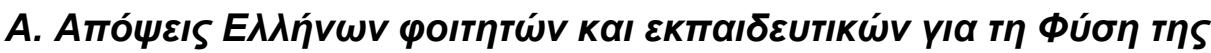

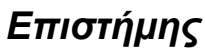

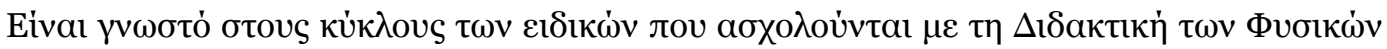

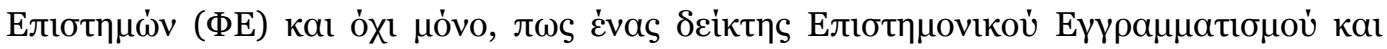

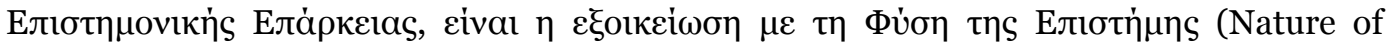

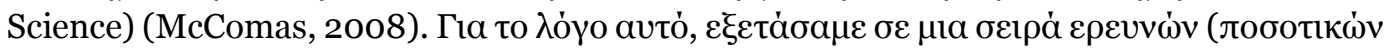




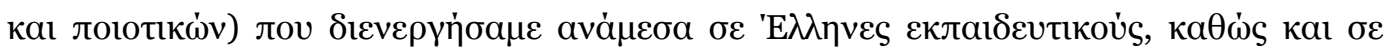

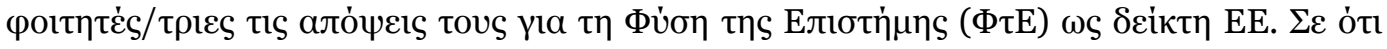

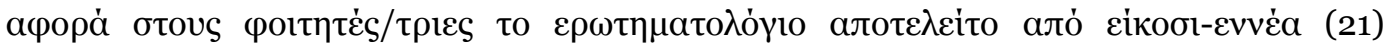

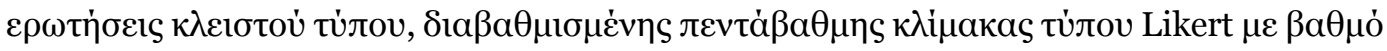

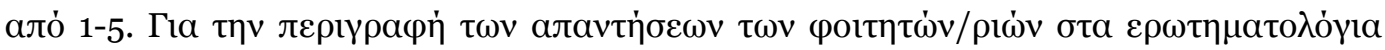

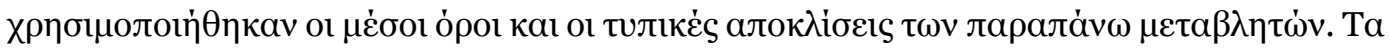

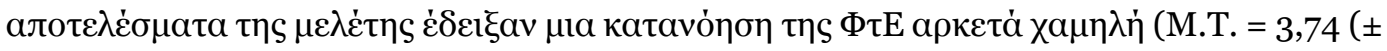
0,40) (Пivakas 1).

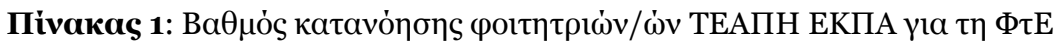

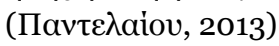

\begin{tabular}{|c|c|}
\hline & 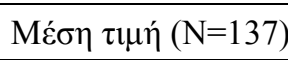 \\
\hline 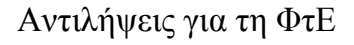 & $3,74+0.40$ \\
\hline
\end{tabular}

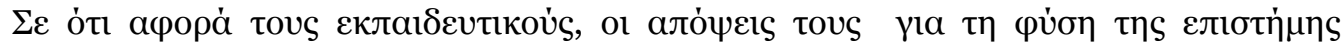

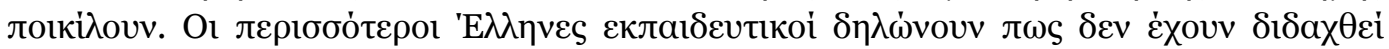

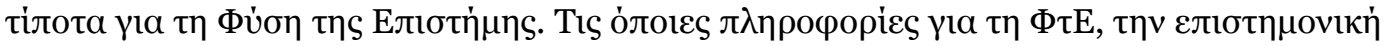

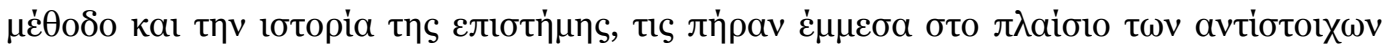

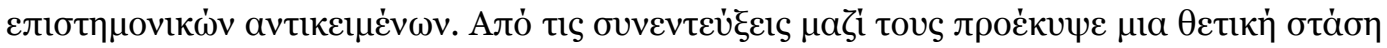

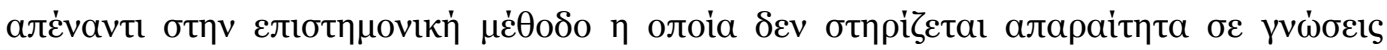

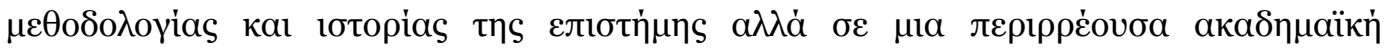

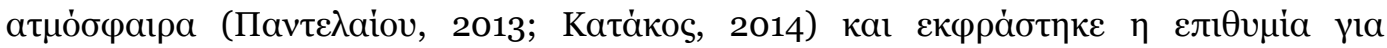

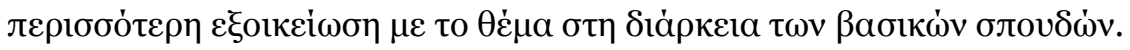

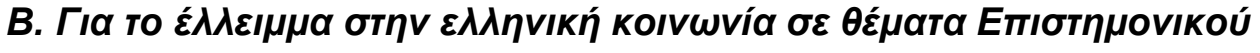

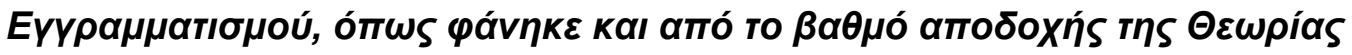

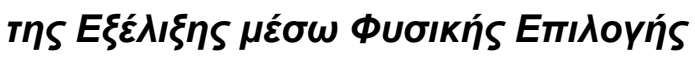

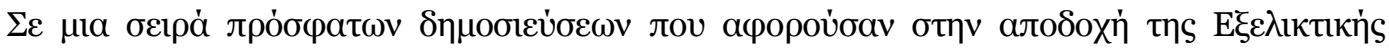

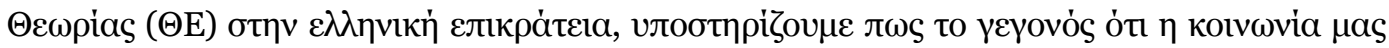

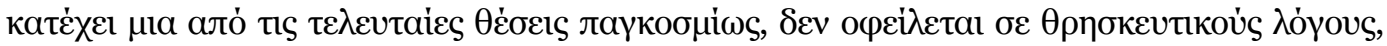

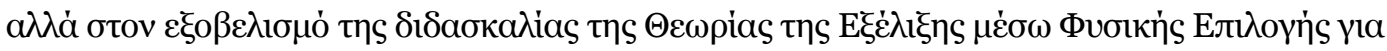

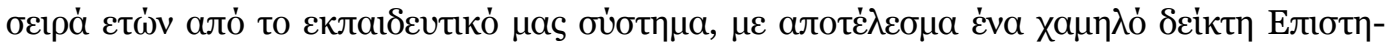

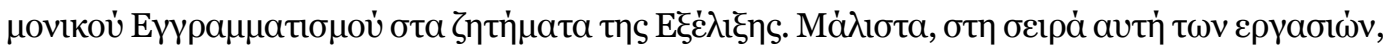

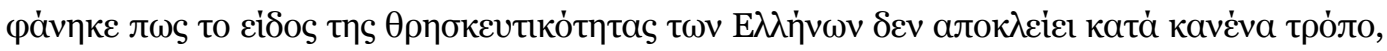

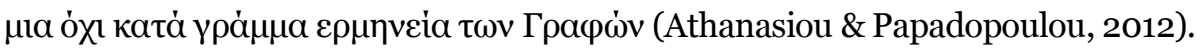




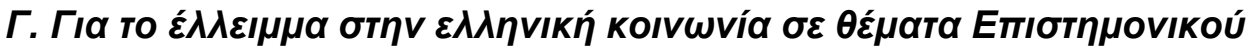

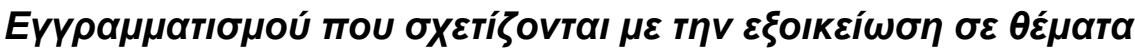

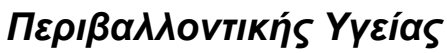

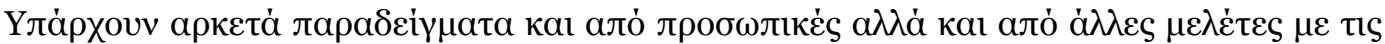

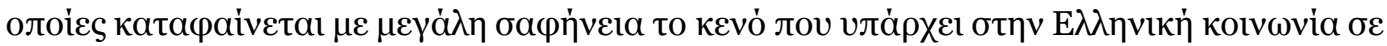

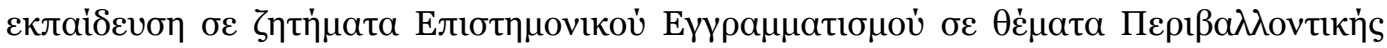

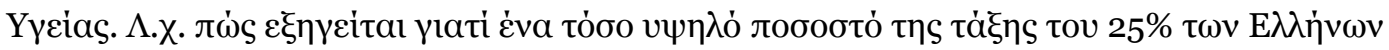

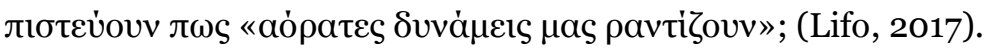

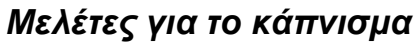

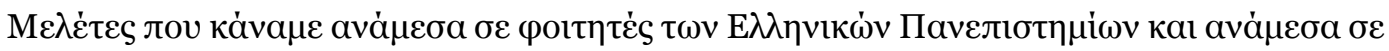

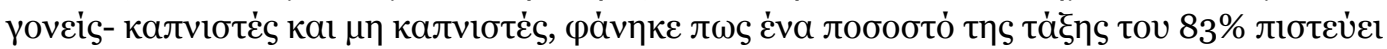

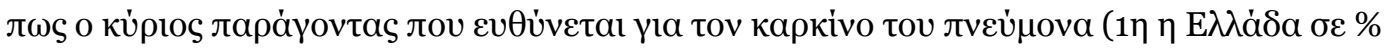

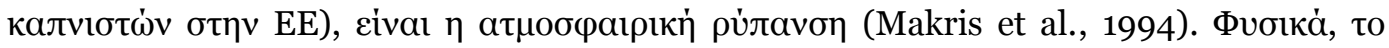

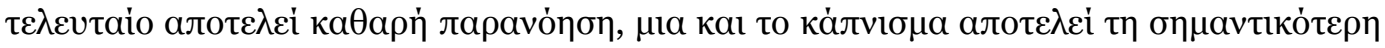

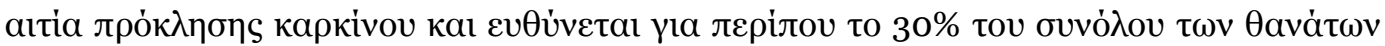

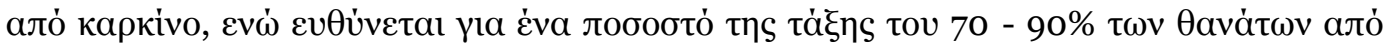

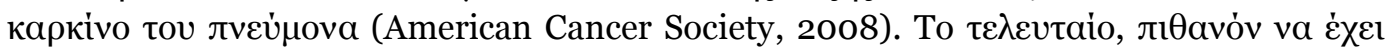

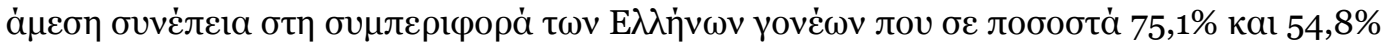

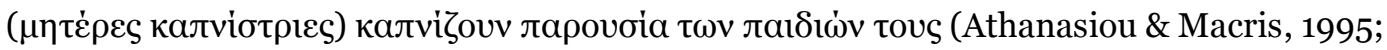

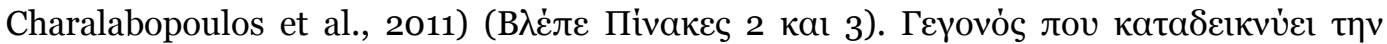

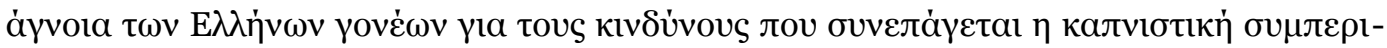

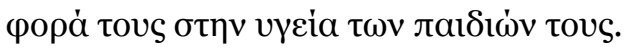

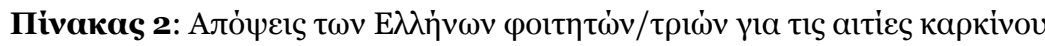

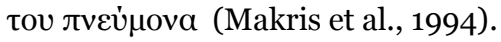

\begin{tabular}{|c|c|c|c|}
\hline \multirow{2}{*}{ 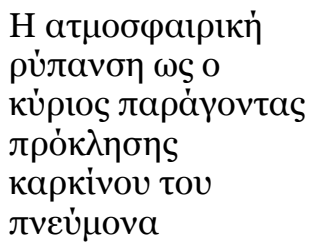 } & 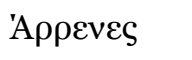 & $\Theta \dot{\eta} \lambda \varepsilon ı 1 \varsigma$ & $\Sigma \dot{v} v o \lambda o$ \\
\hline & n \% & n \% & n $\%$ \\
\hline $\mathrm{Nal}$ & 39082.1 & 54983.0 & 93982.7 \\
\hline 'Oxl & $23 \quad 4.8$ & 91.4 & 322.8 \\
\hline$\Delta \varepsilon v \gamma v \omega \rho i \zeta \omega$ & 6213.1 & 10315.6 & 16514.5 \\
\hline 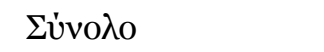 & 475100.0 & 661100.0 & 1136100.0 \\
\hline
\end{tabular}




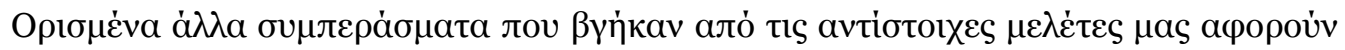

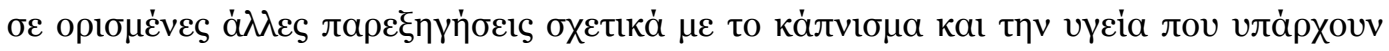

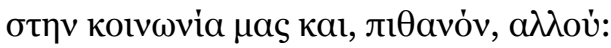

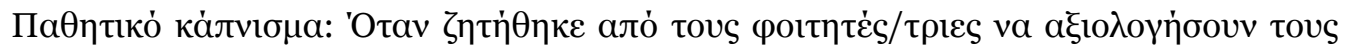

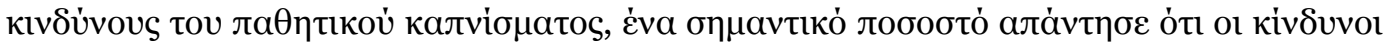

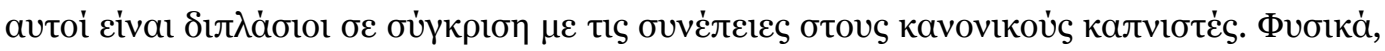

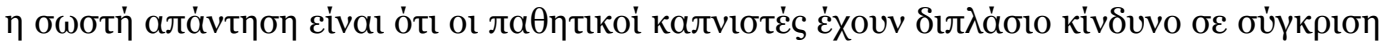

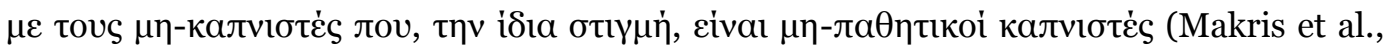
1994).

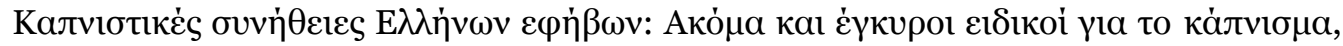

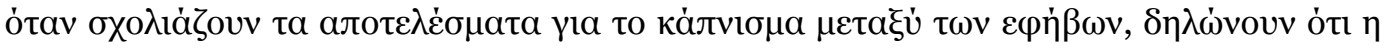

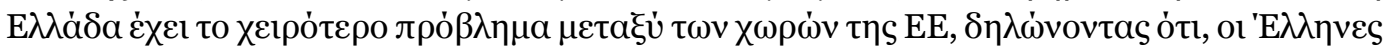

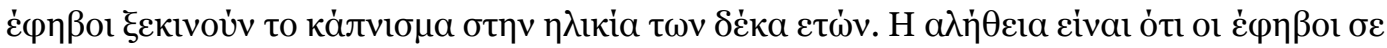

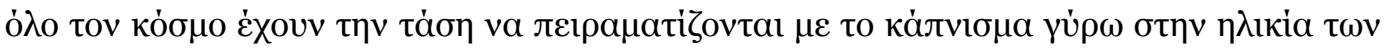

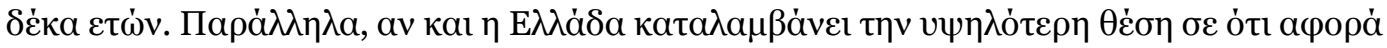

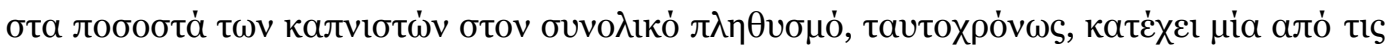

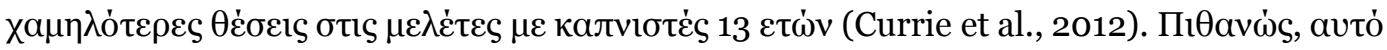

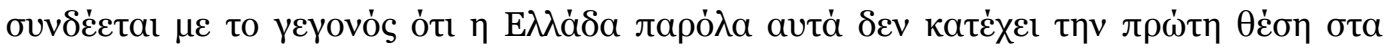

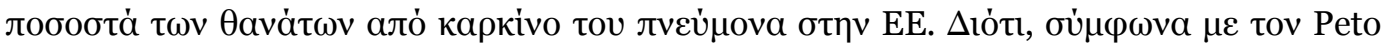

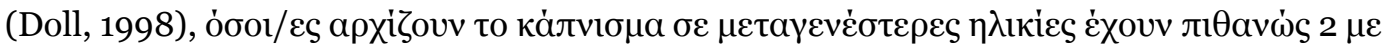

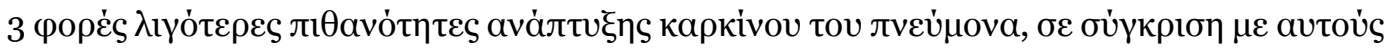

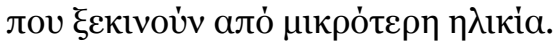

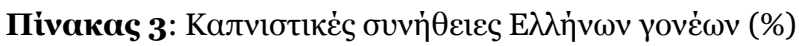

(Charalabopoulos et al., 2011)

\begin{tabular}{|c|c|c|c|}
\hline 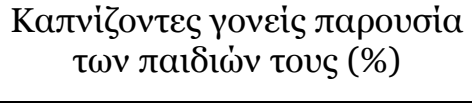 & 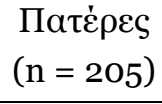 & 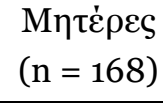 & $\begin{array}{c}\sum \dot{v} v 0 \lambda \mathrm{o} \\
(\mathrm{n}=373)\end{array}$ \\
\hline $\mathrm{Nal}$ & 75.1 & 54.8 & 66.0 \\
\hline 'OХl & 11.2 & 12.5 & 11.8 \\
\hline 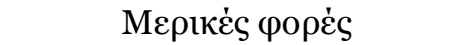 & 13.7 & 32.7 & 22.2 \\
\hline
\end{tabular}

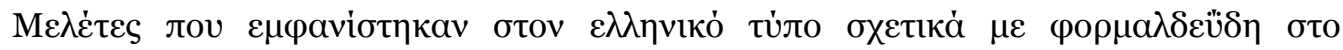

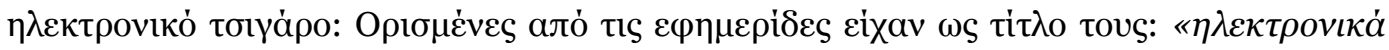

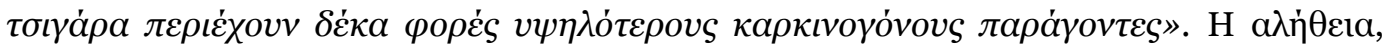

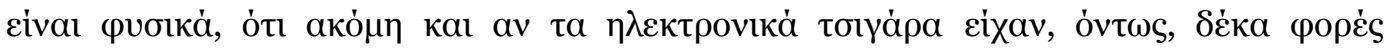

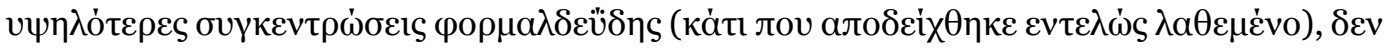

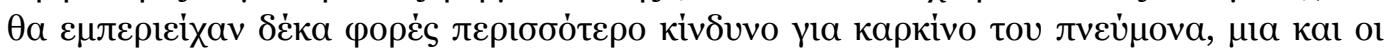




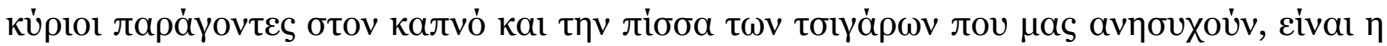

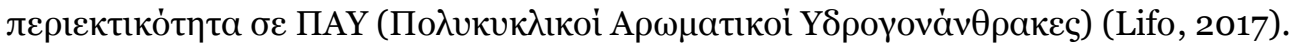

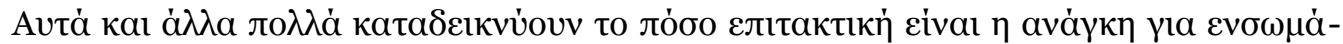

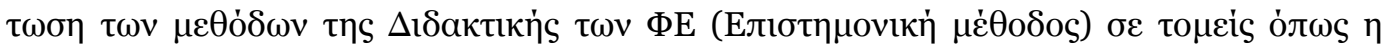

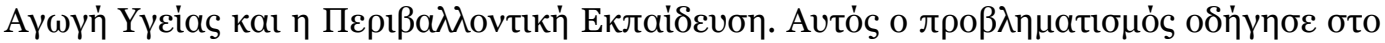

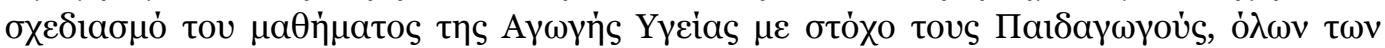

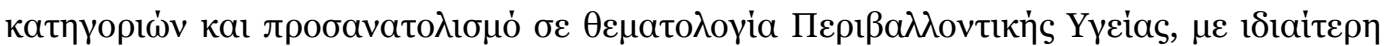

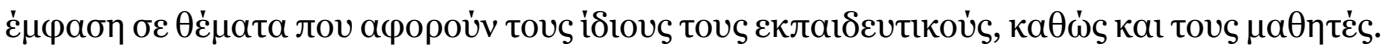

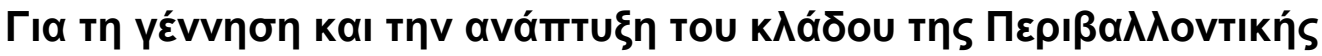

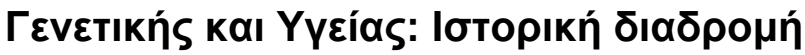

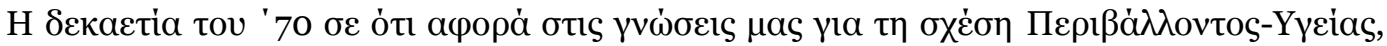

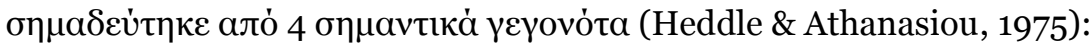

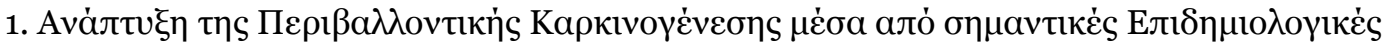

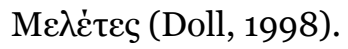

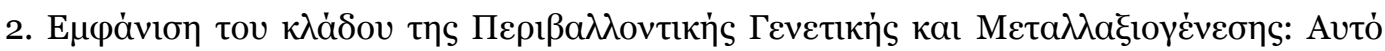

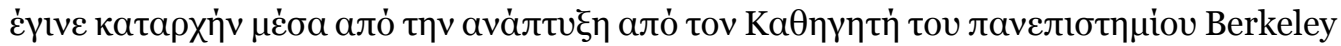

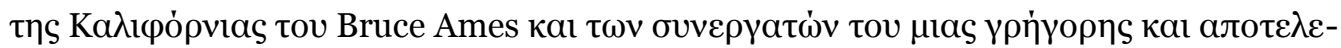

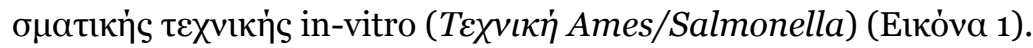

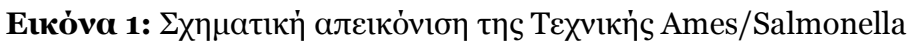

\section{The Ames/Salmonella system}

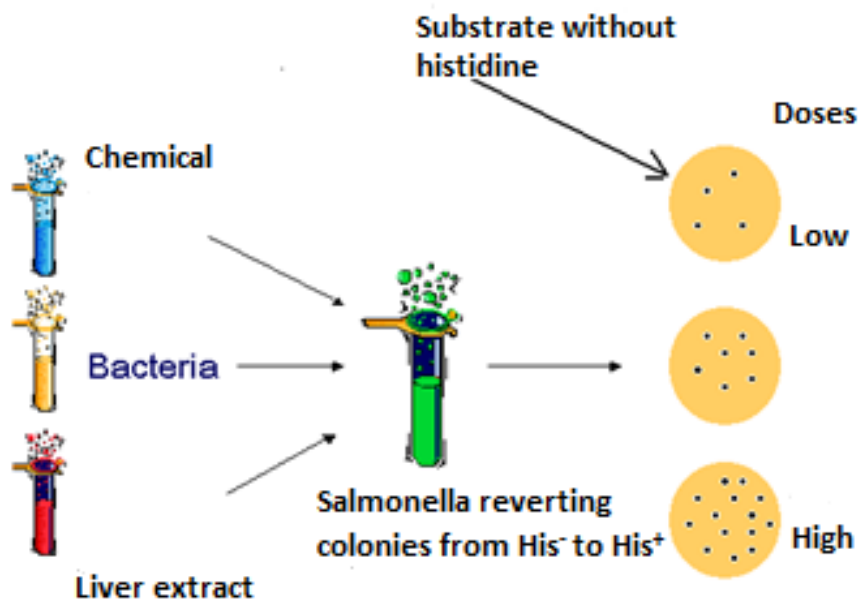




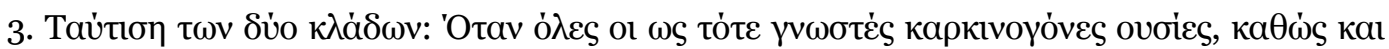

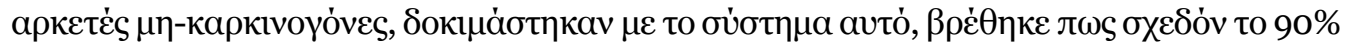

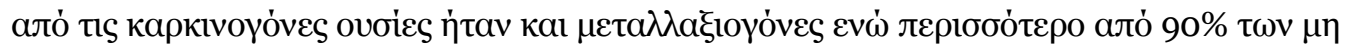

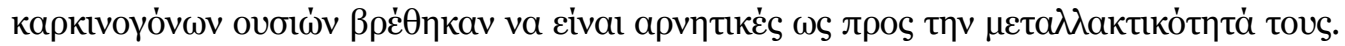

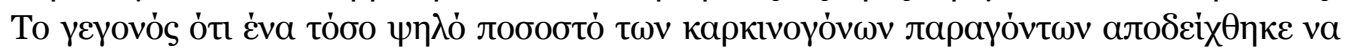

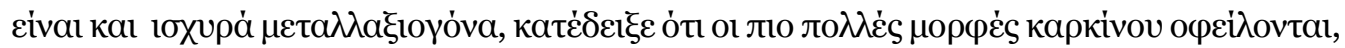

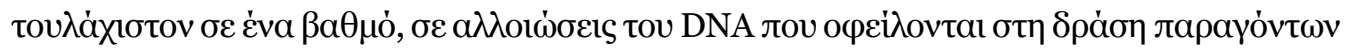

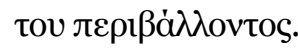

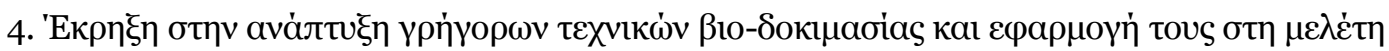

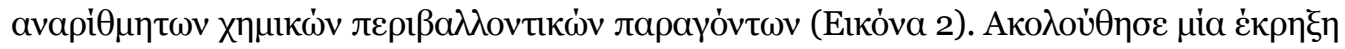

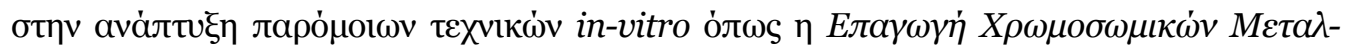

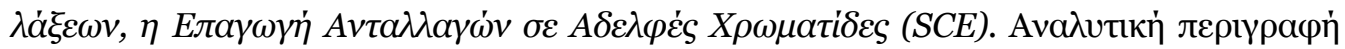

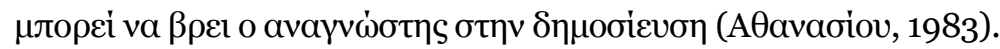

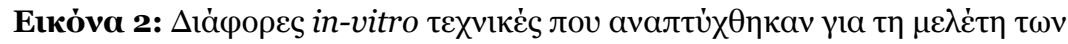

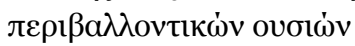

\section{Chromosome aberrations}

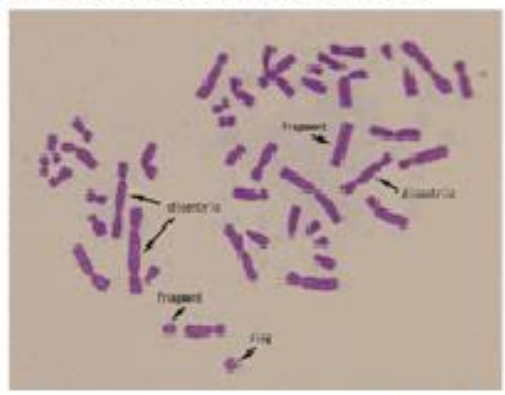

Sister Chromatid Exchanges

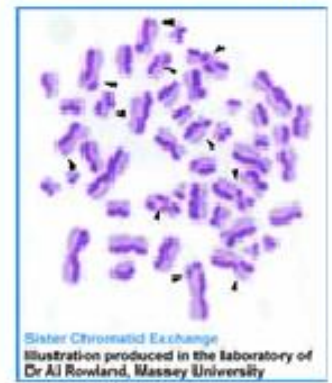

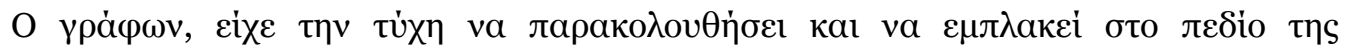

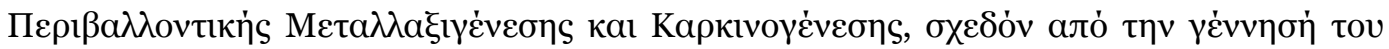

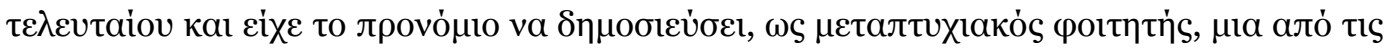

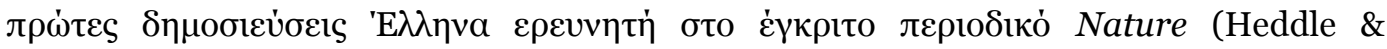

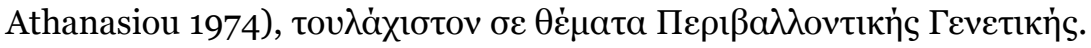

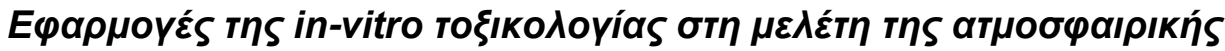

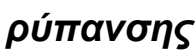

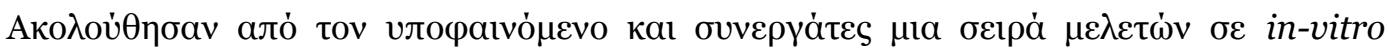

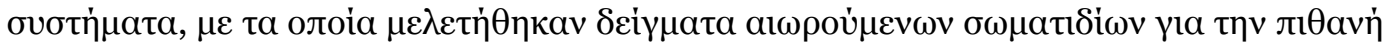

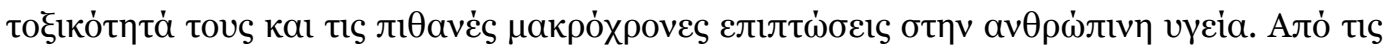

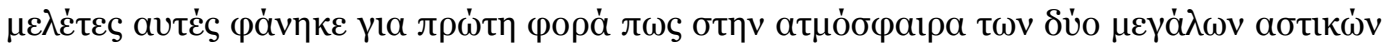




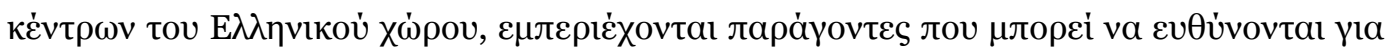

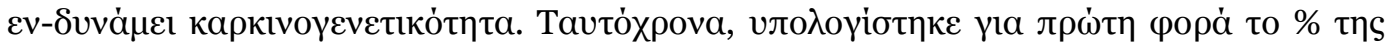

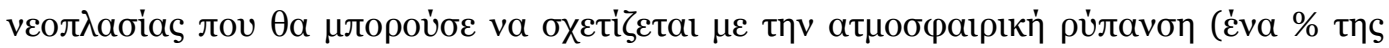

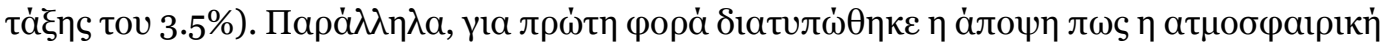

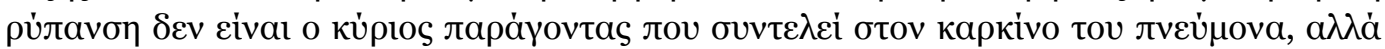

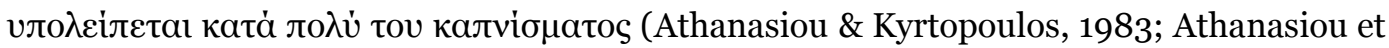
al., 1987).

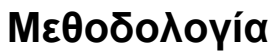

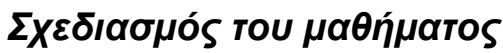

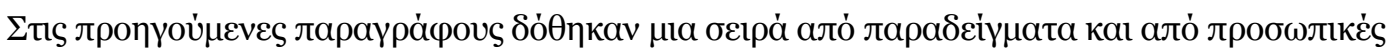

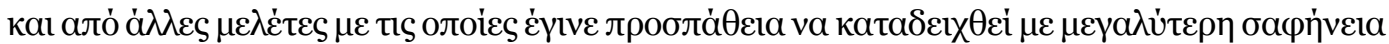

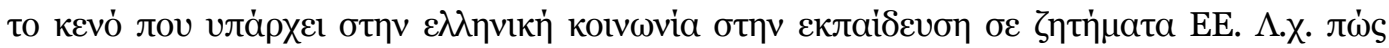

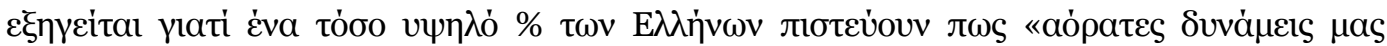

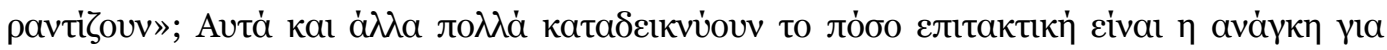

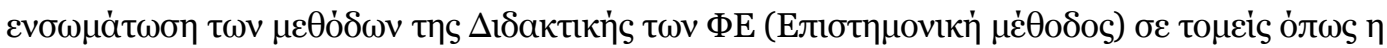

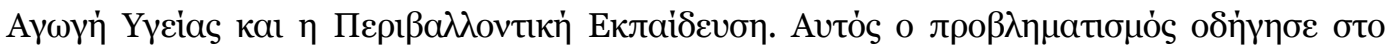

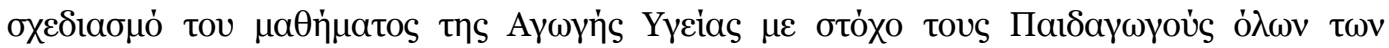

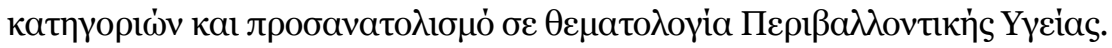

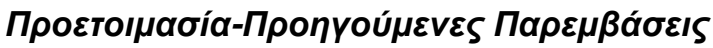

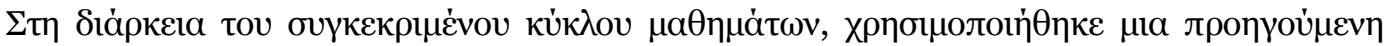

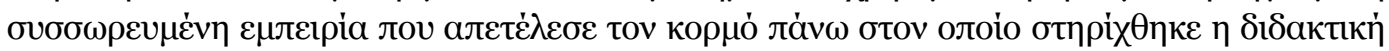

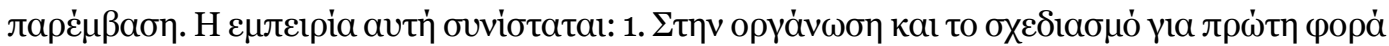

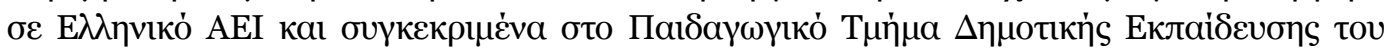

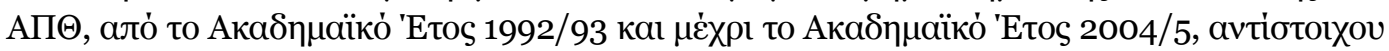

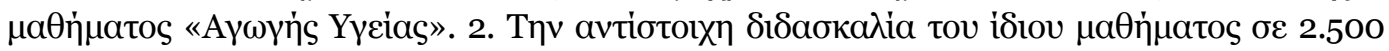

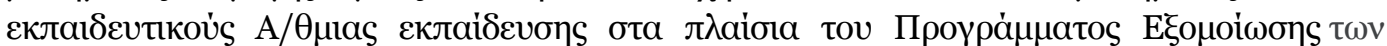

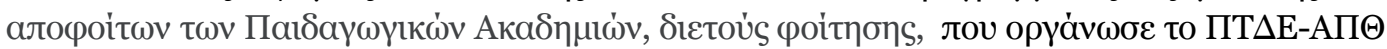

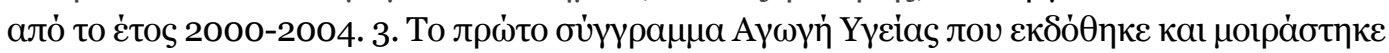

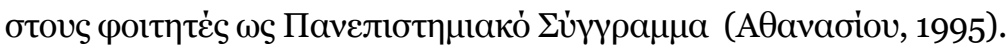

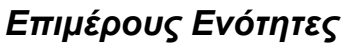

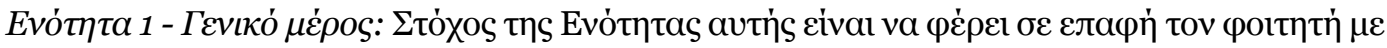

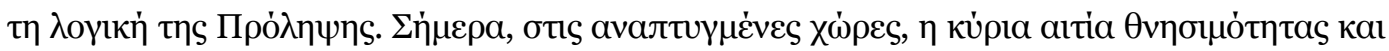

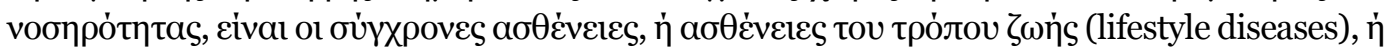

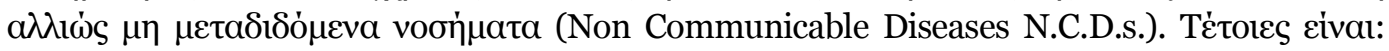




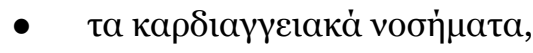

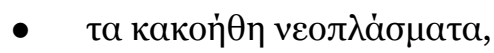

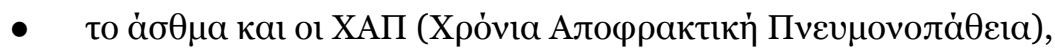

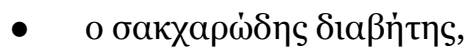

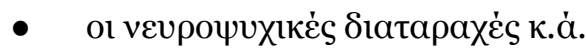

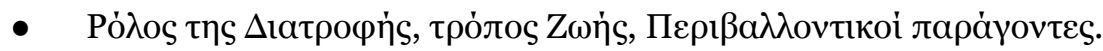

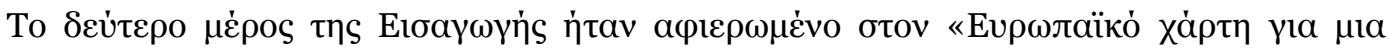

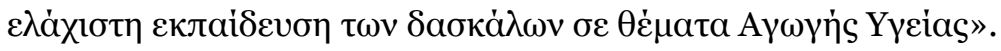

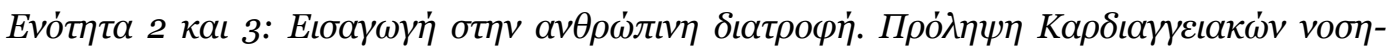

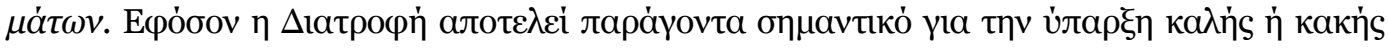

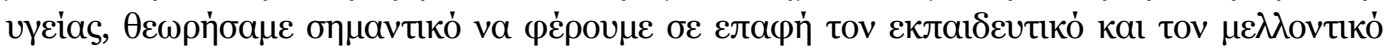

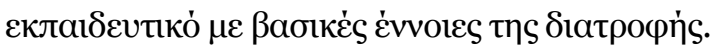

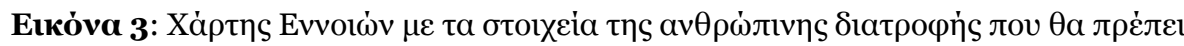

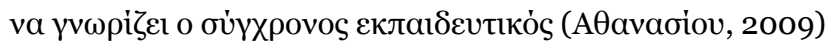
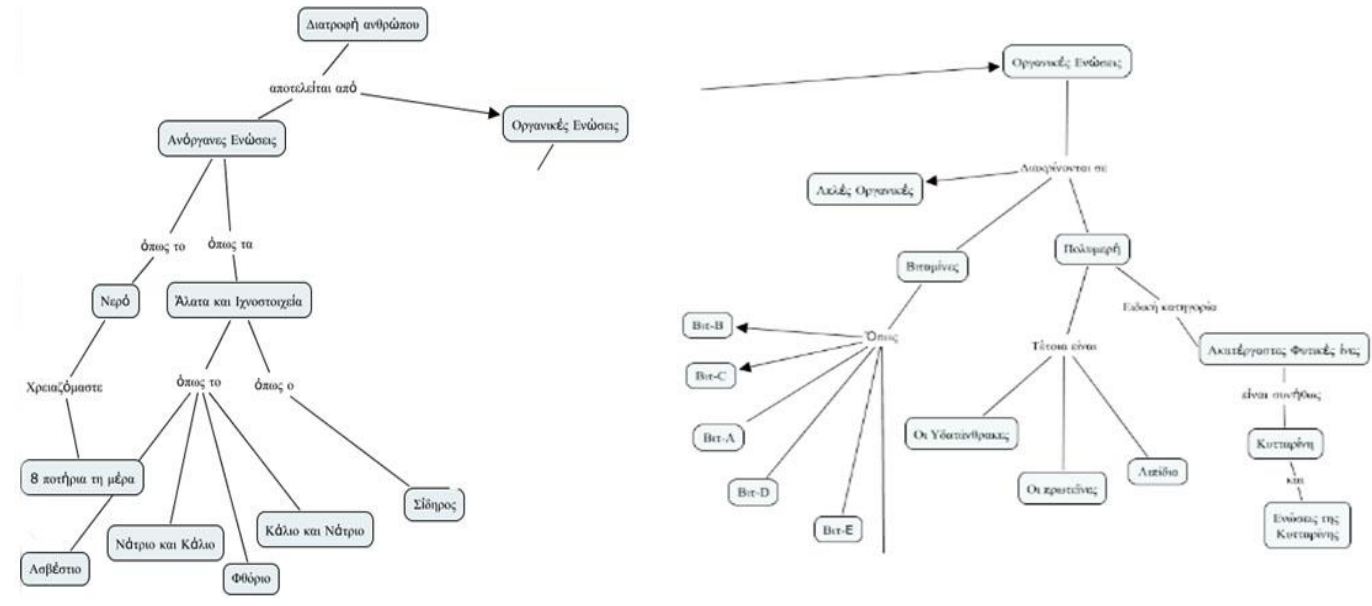

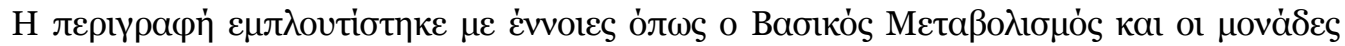

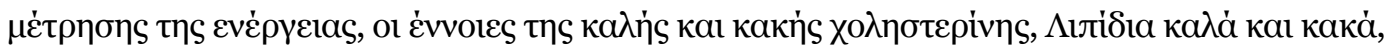

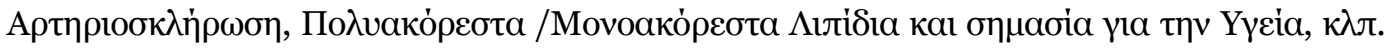

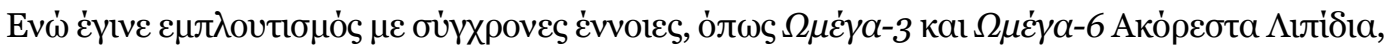

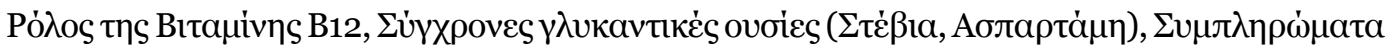

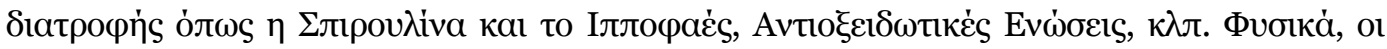

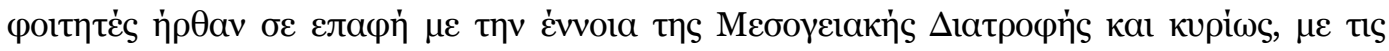




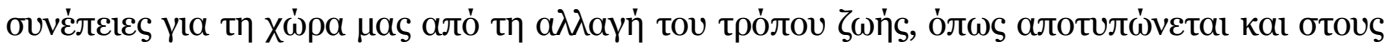

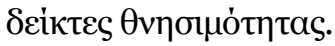

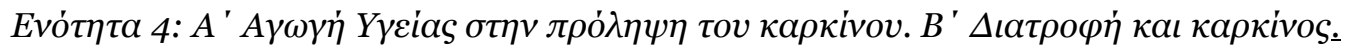

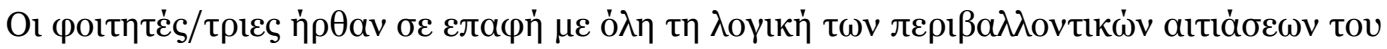

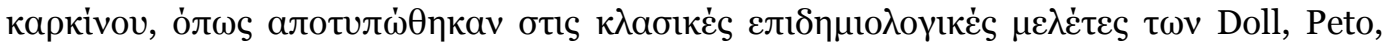

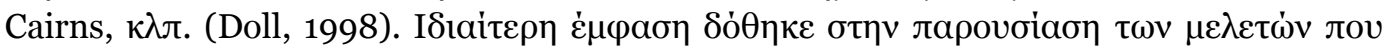

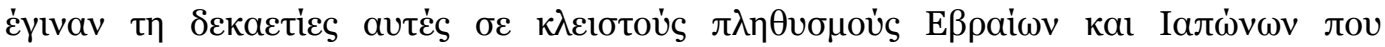

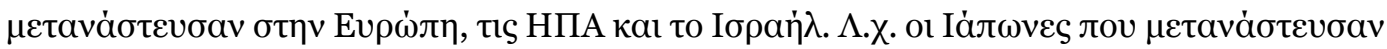

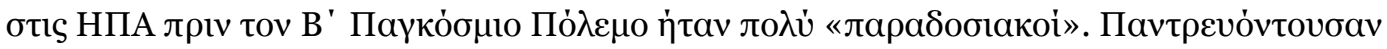

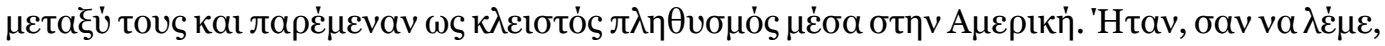

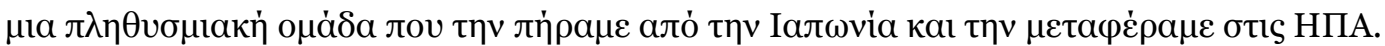

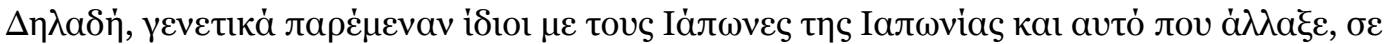

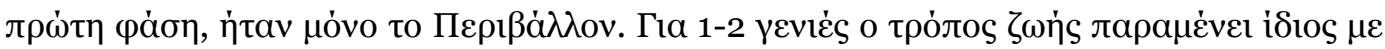

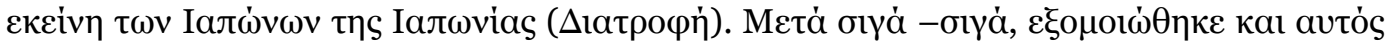

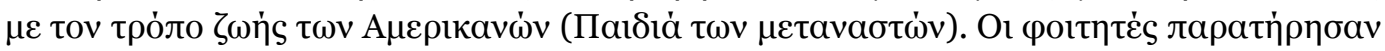

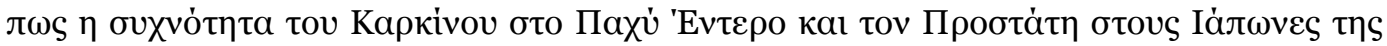

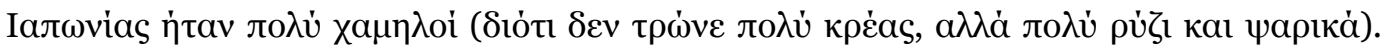

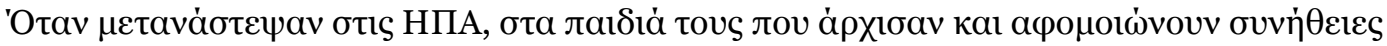

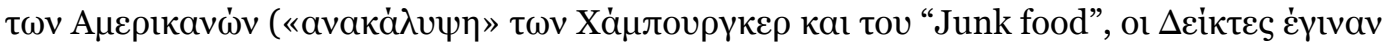

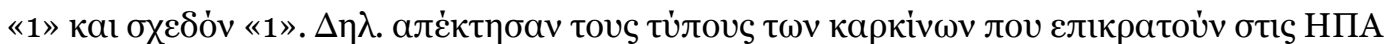

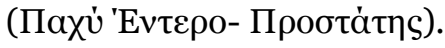

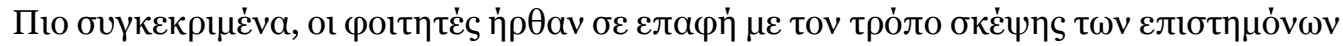

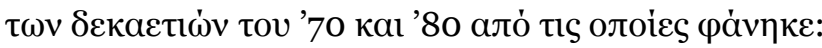

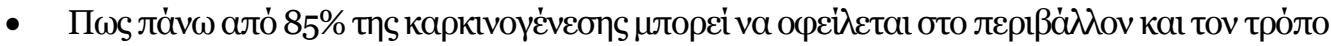

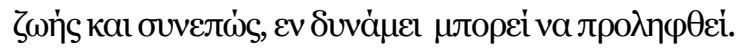

- 'H

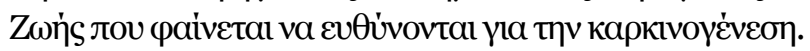

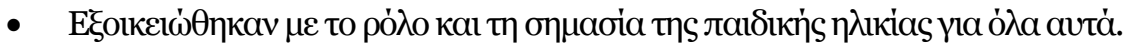

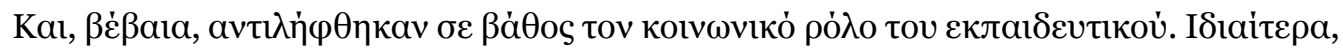

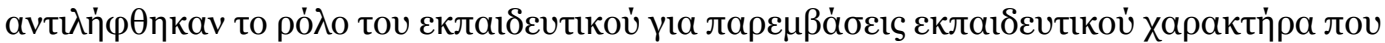

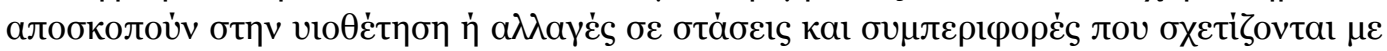

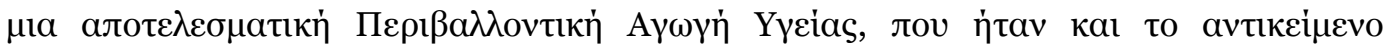

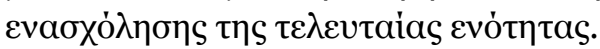

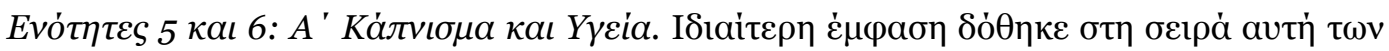

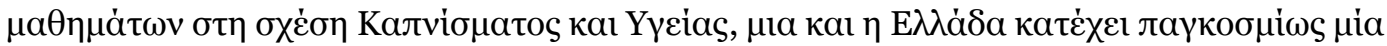

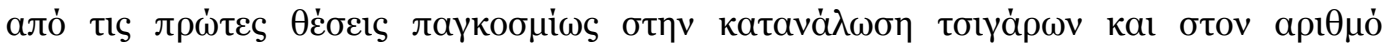

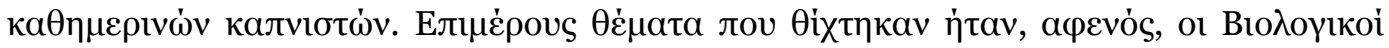

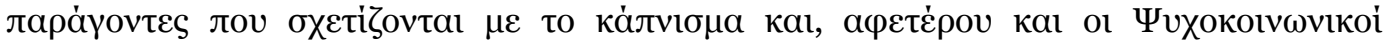

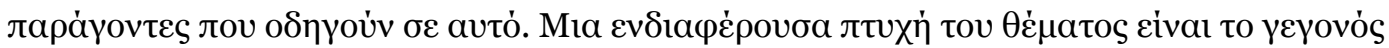




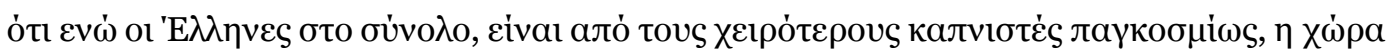

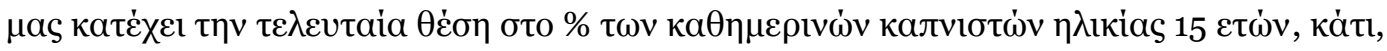

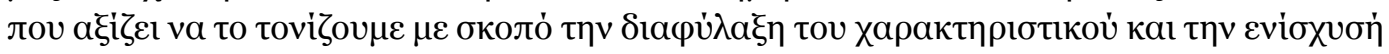
tov.

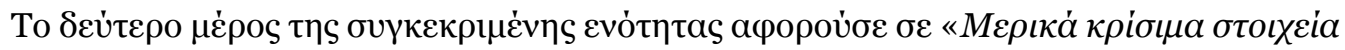

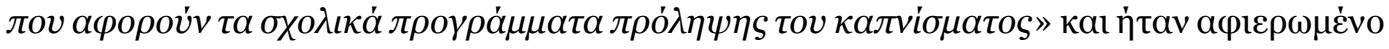

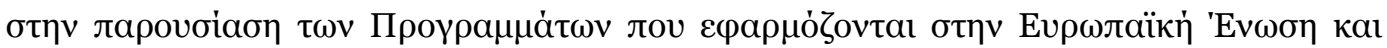

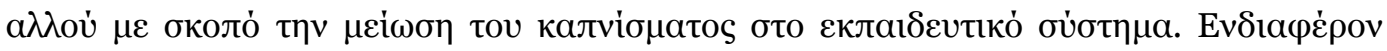

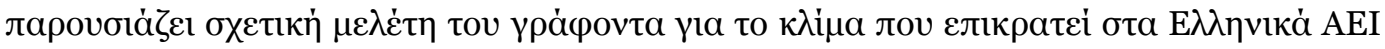

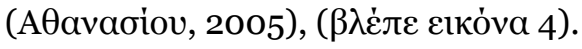

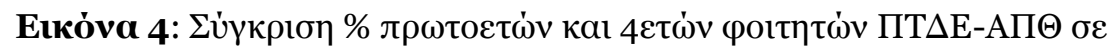

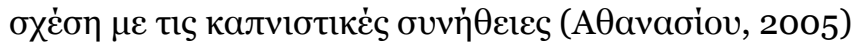

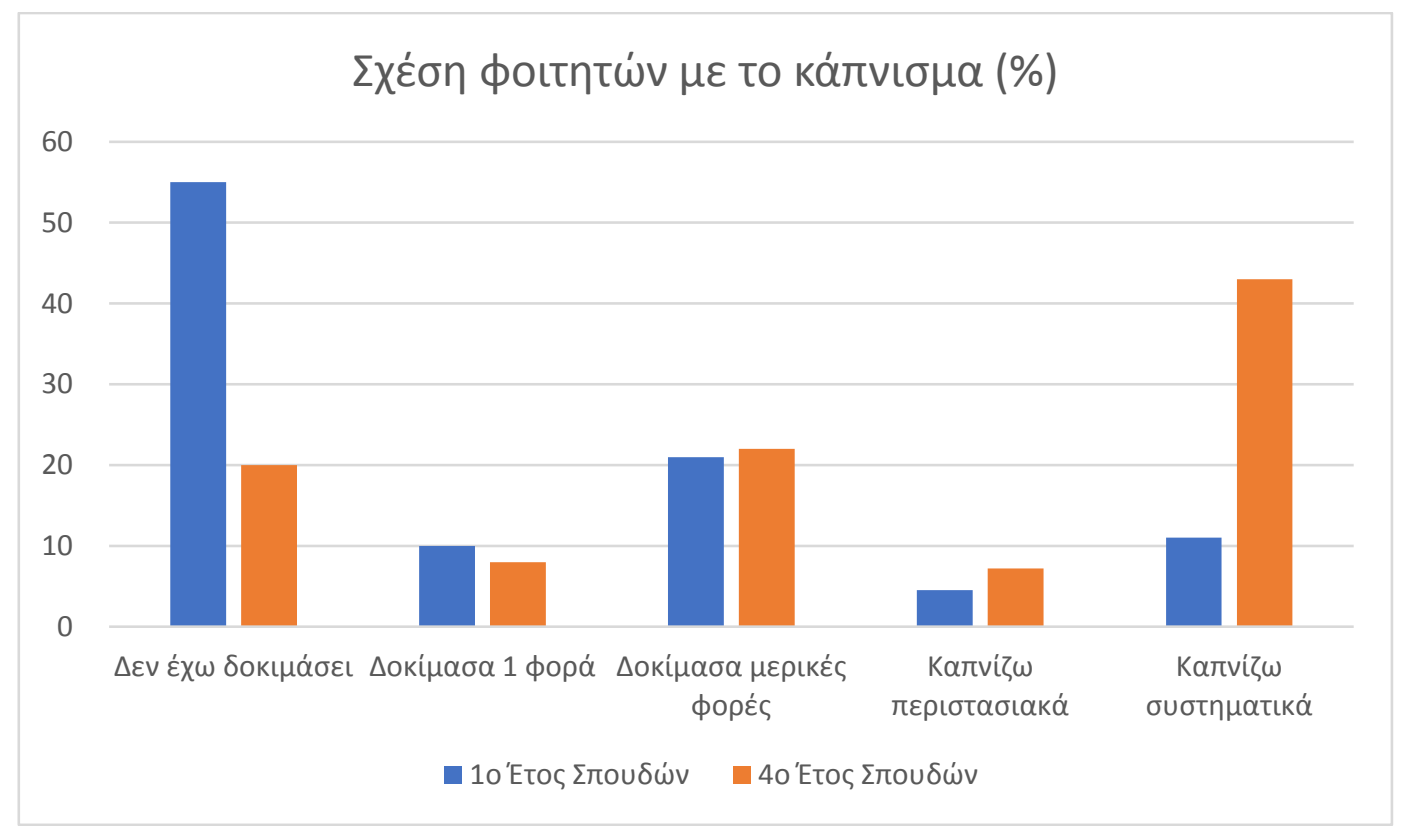

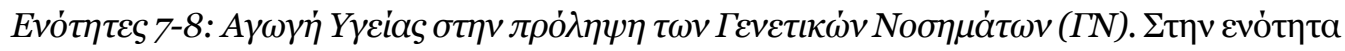

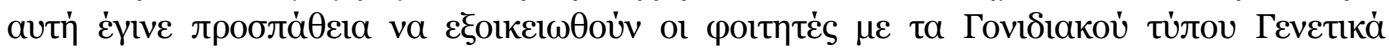

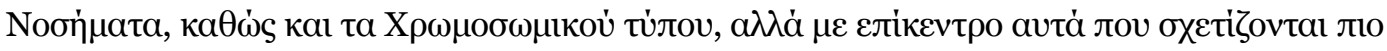

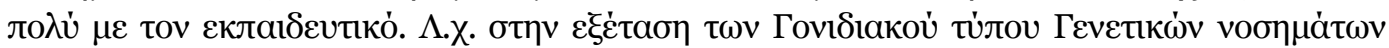

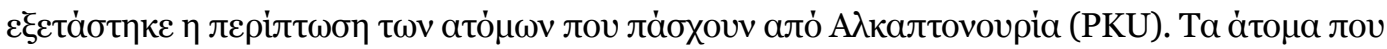

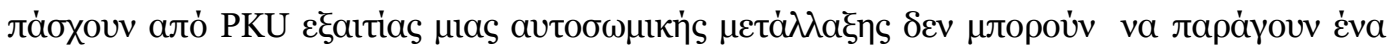
$\dot{\varepsilon} v \zeta$

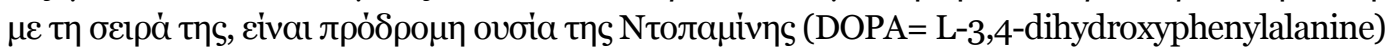




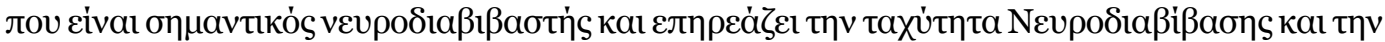

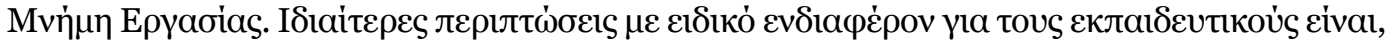

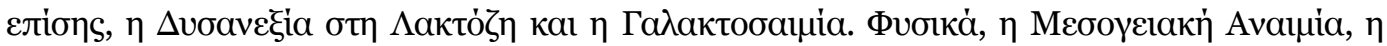

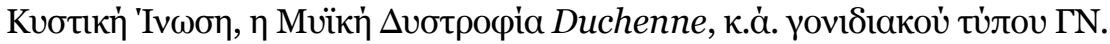

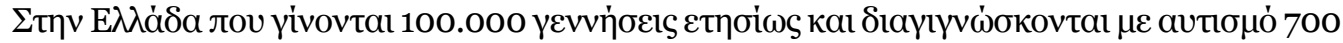

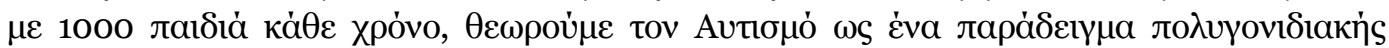

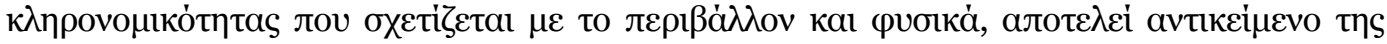

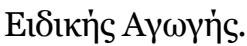

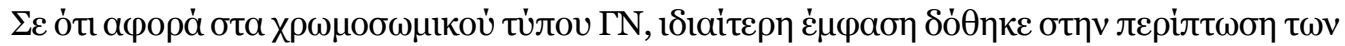

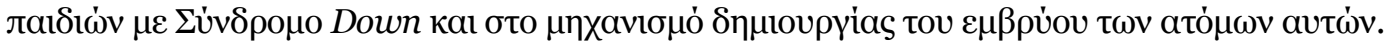

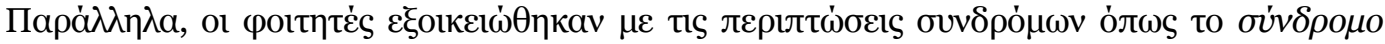

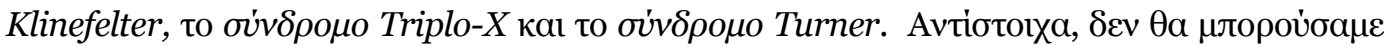

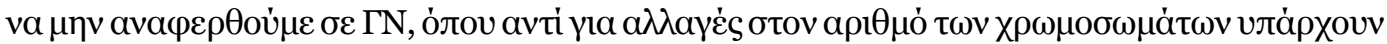

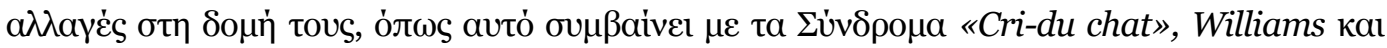

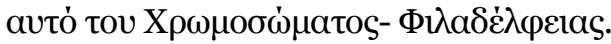

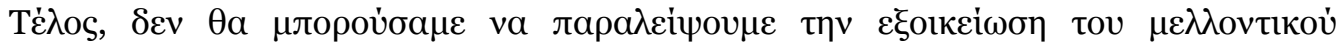

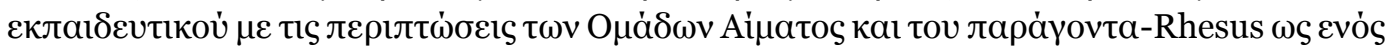

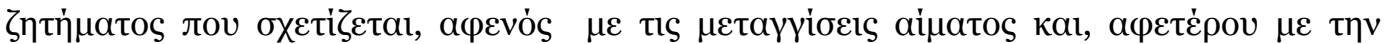

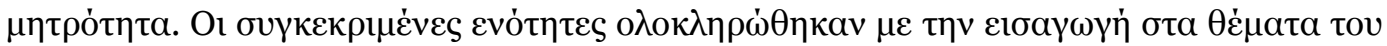

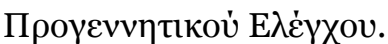

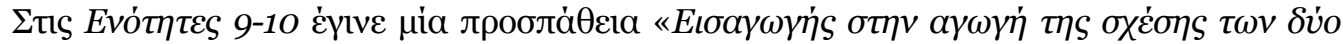

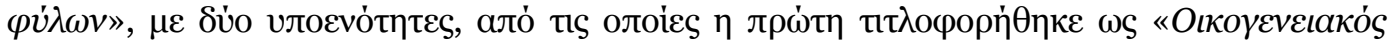

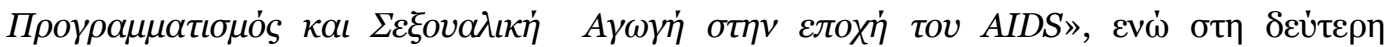

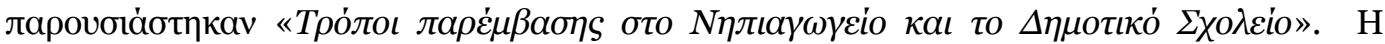

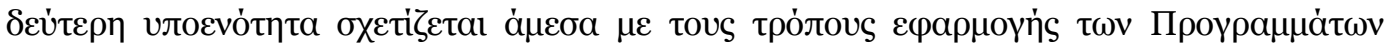

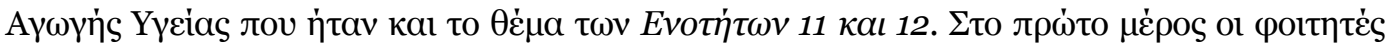

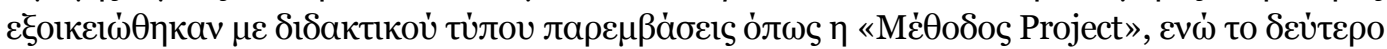

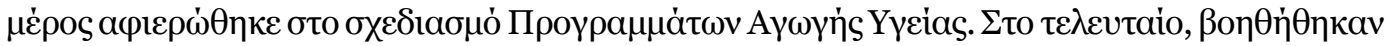

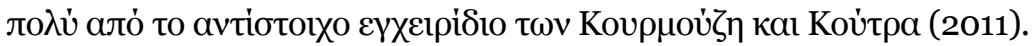

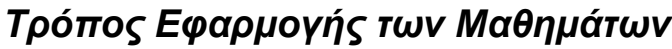

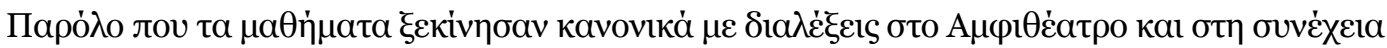

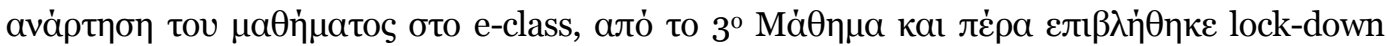

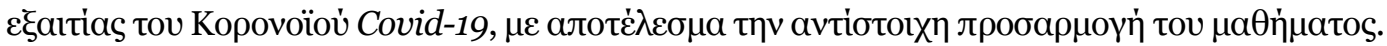

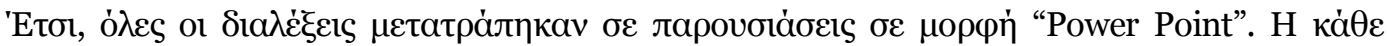

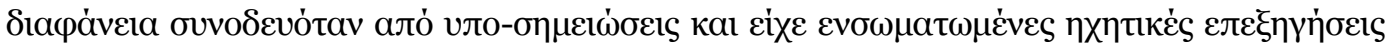

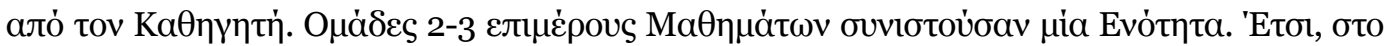

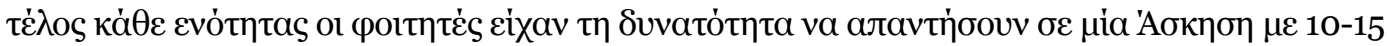

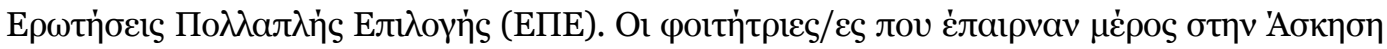




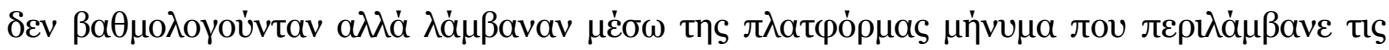

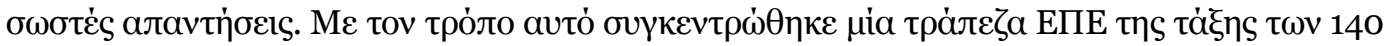

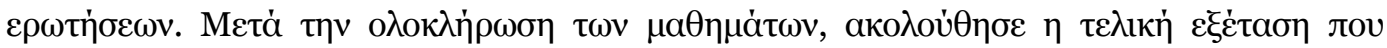

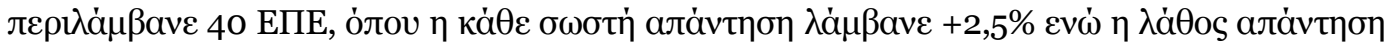

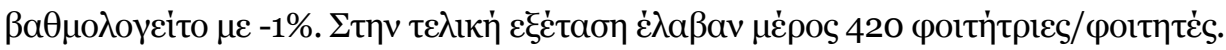

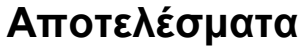

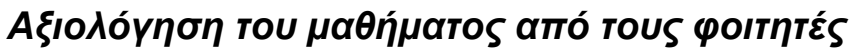

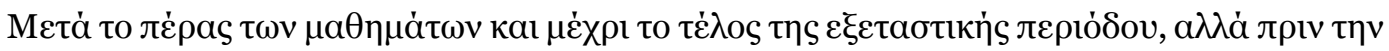

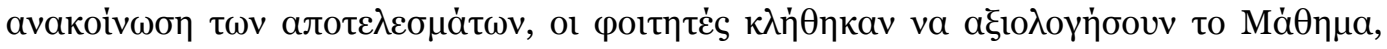

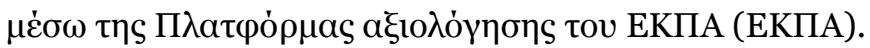

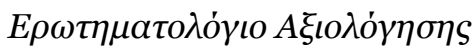

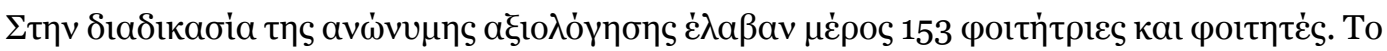

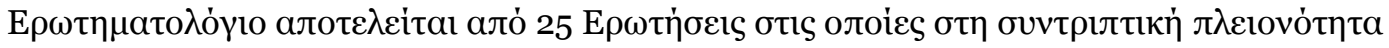

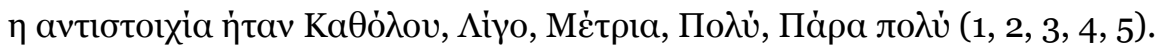

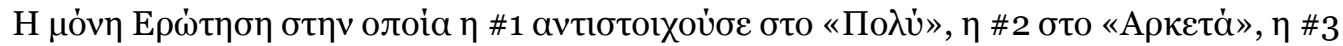

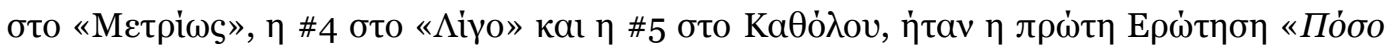

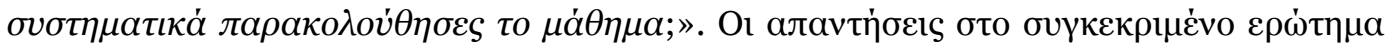

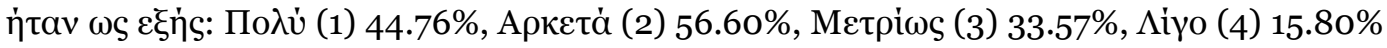

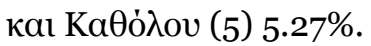

Та ขло்

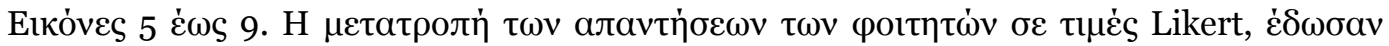

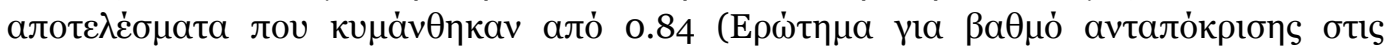

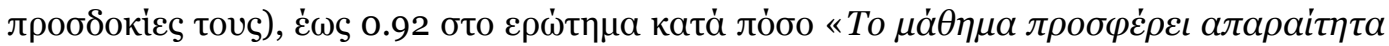

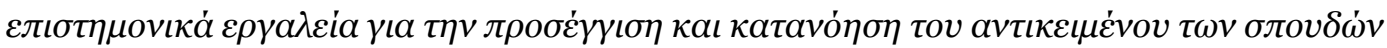
$\sigma \alpha \varsigma \gamma \varepsilon v i \kappa o ́ \tau \varepsilon \rho \alpha »$. 


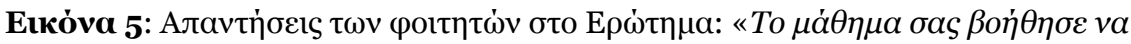

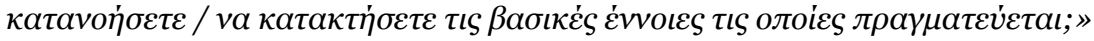

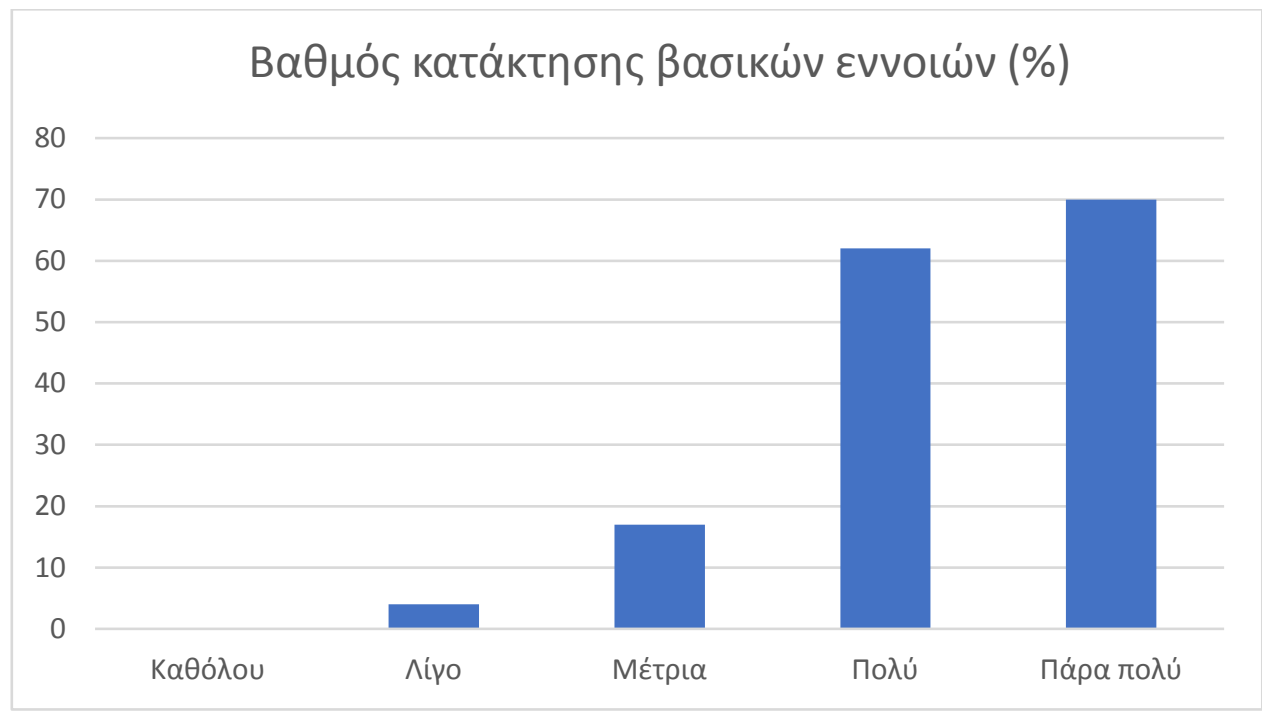

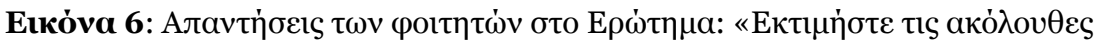

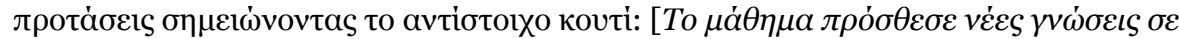

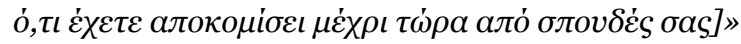

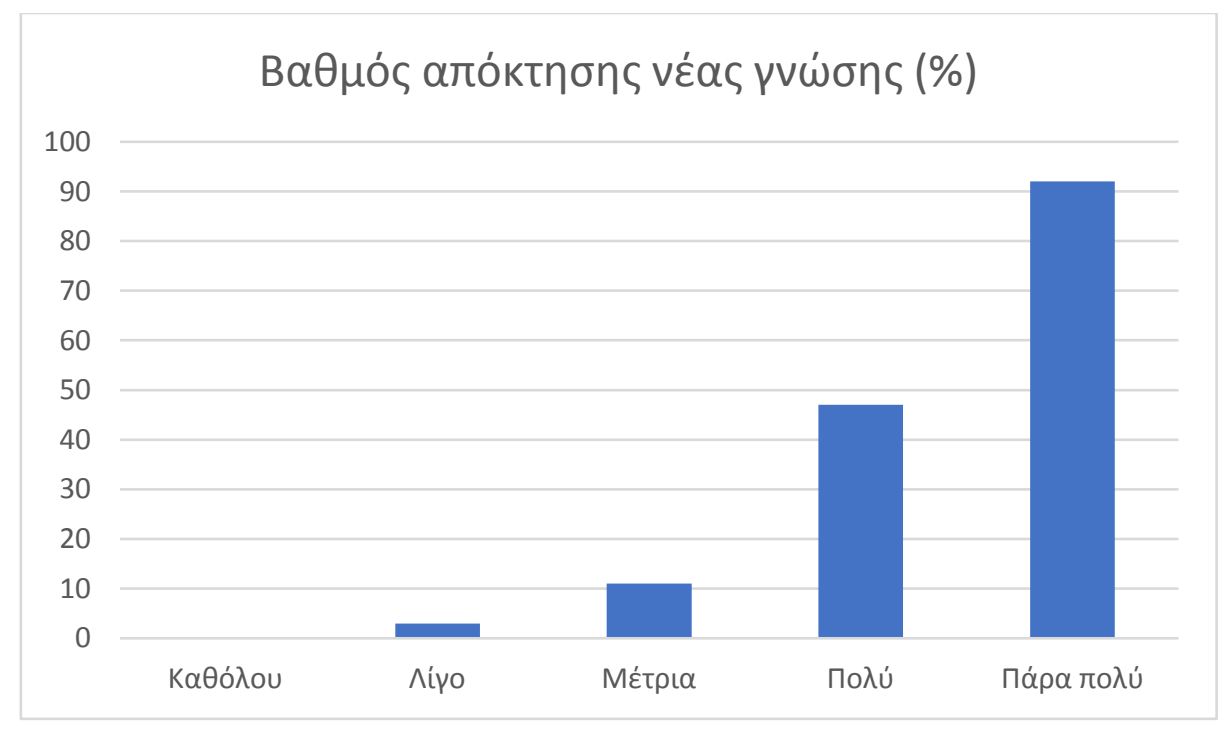




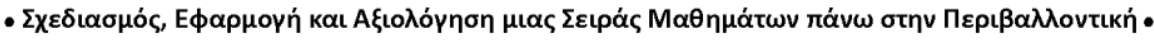

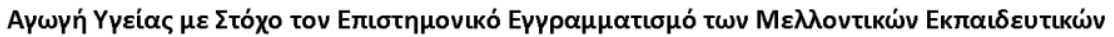

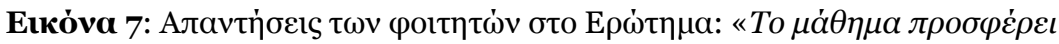

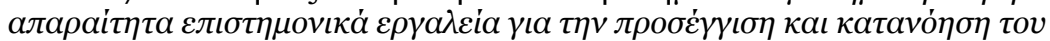

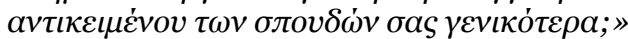

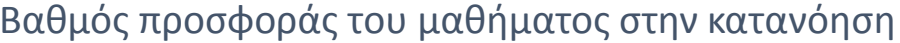

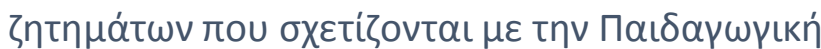

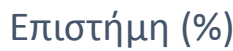

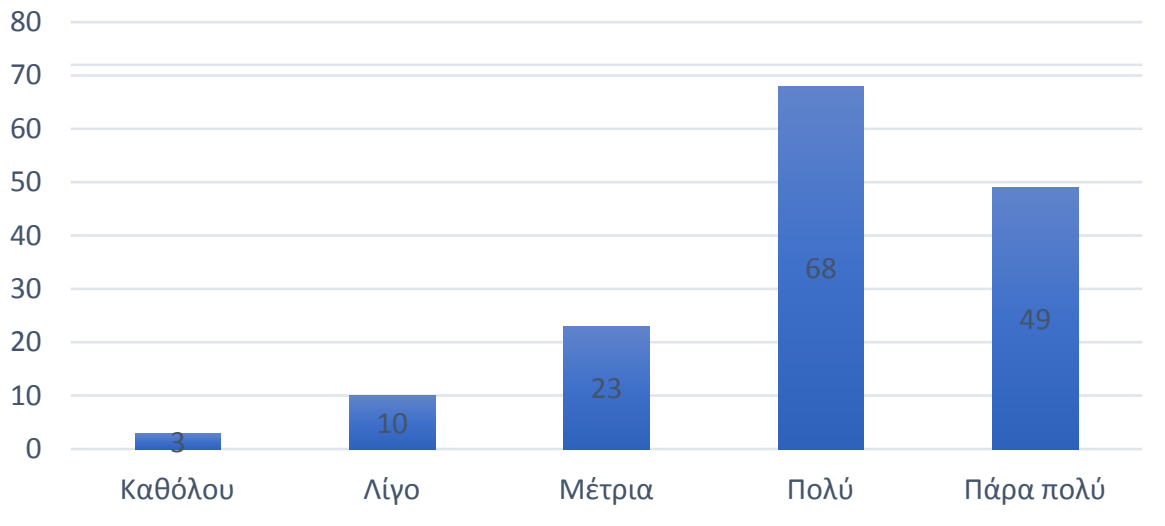

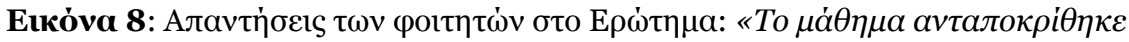

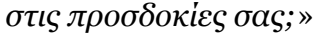

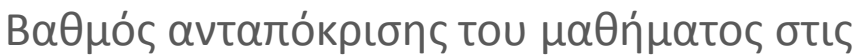 пробокієৎ тоuৎ (\%)}

70

60

50

40

30

20

10

0

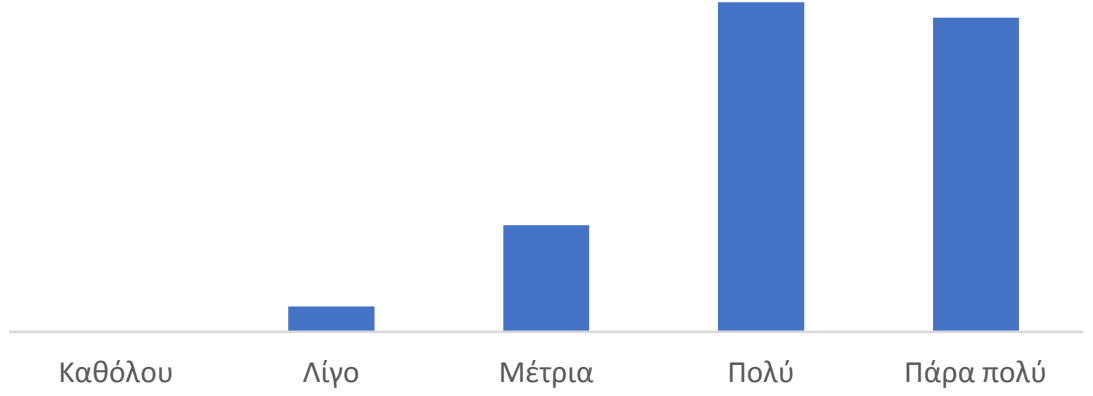




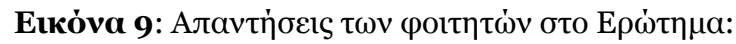

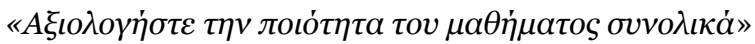

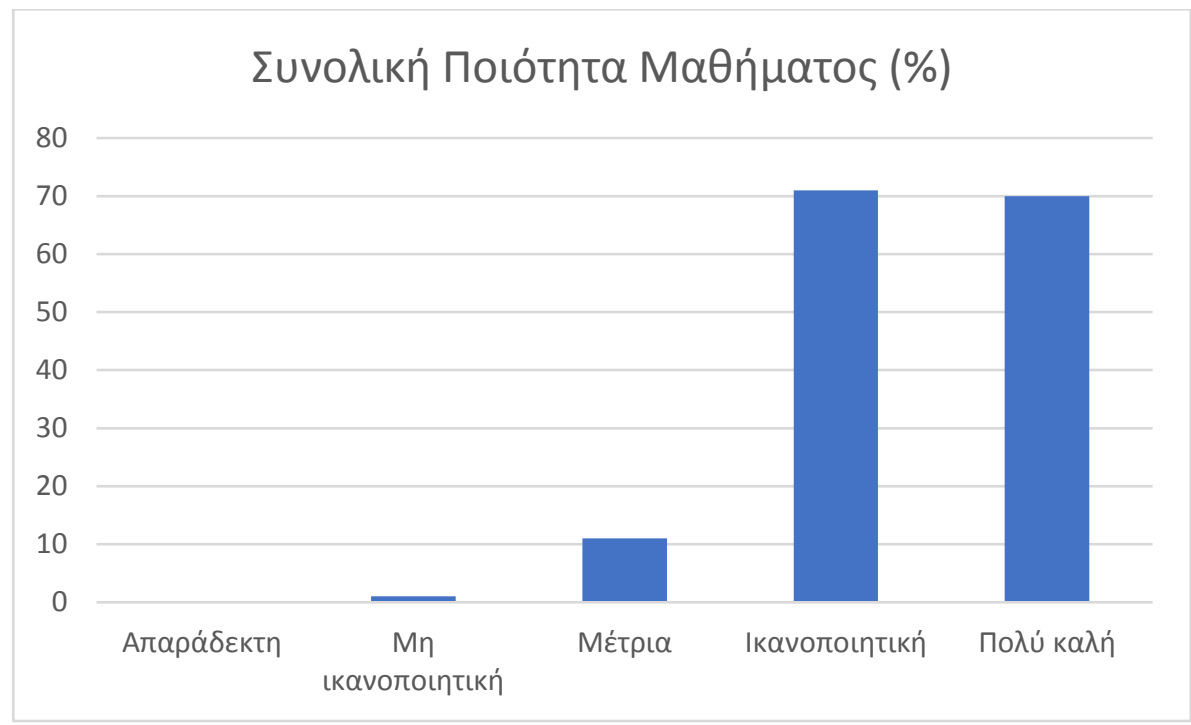

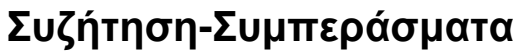

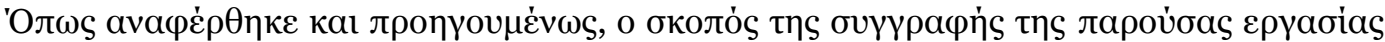

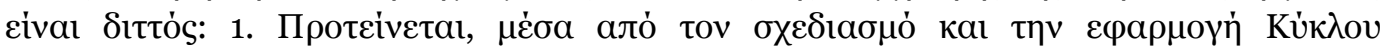

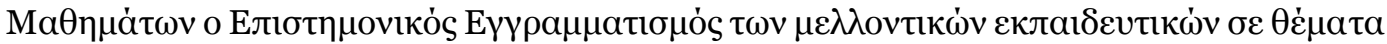

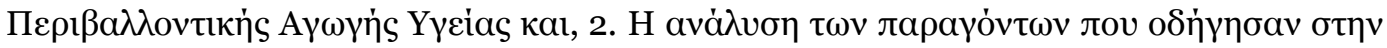

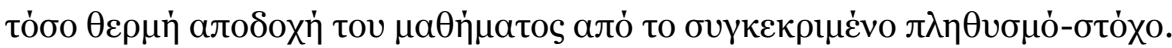

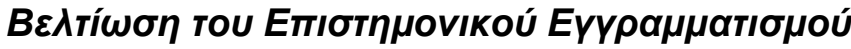

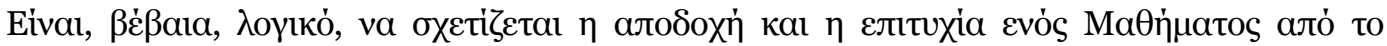

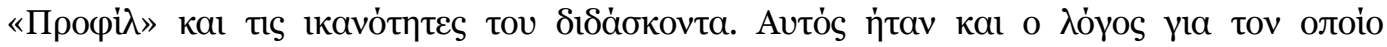

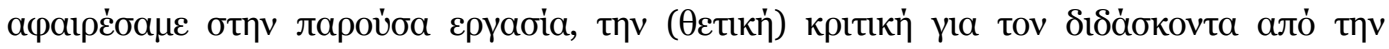

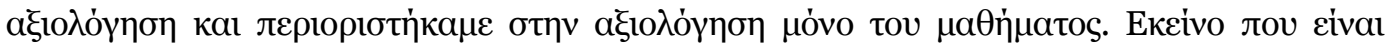

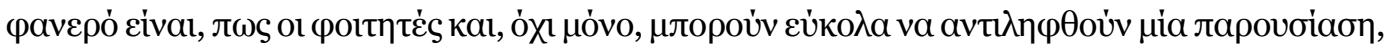

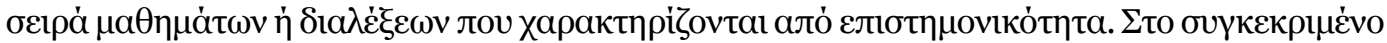

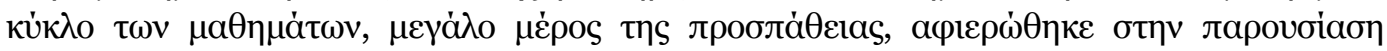

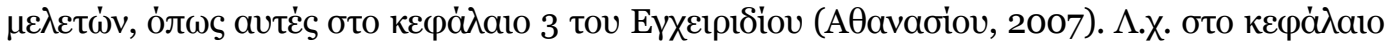

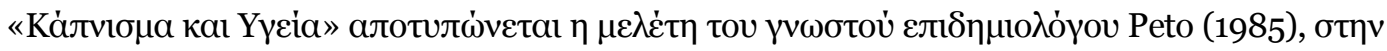

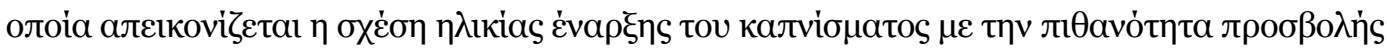




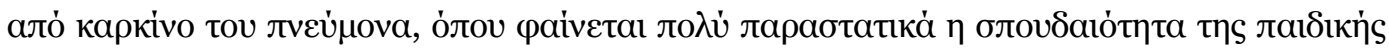

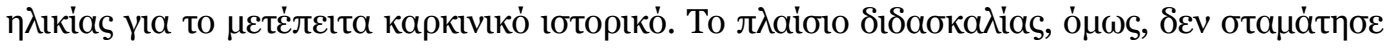

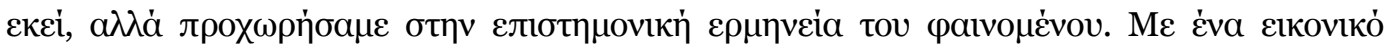

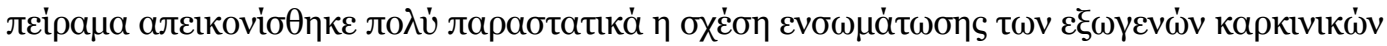

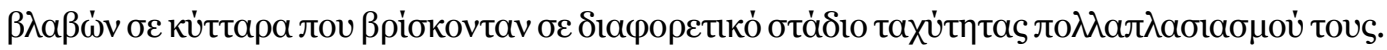

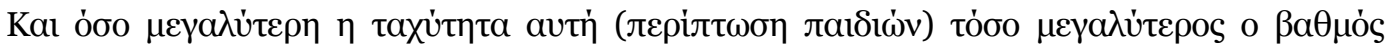

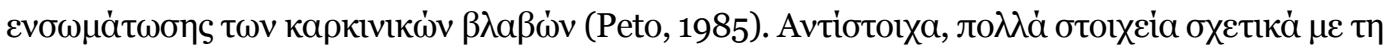

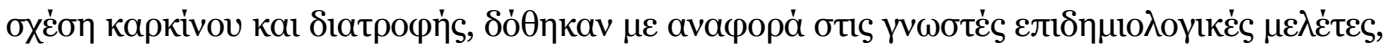

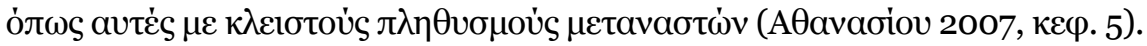

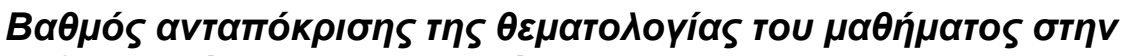

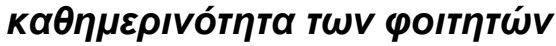

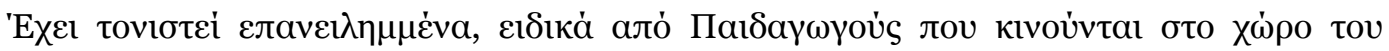

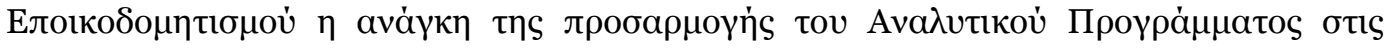

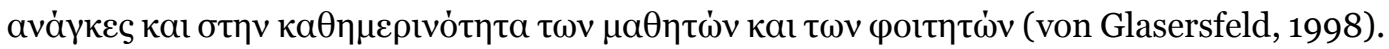

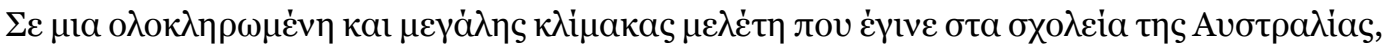

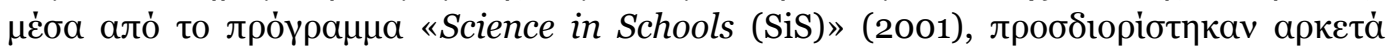

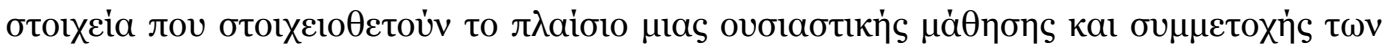

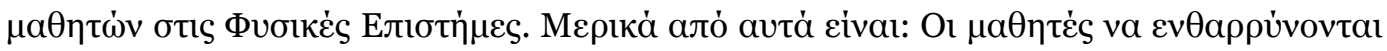

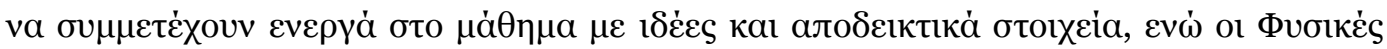

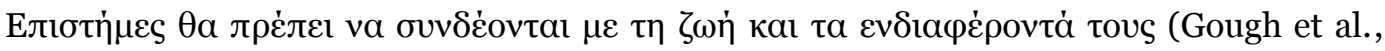

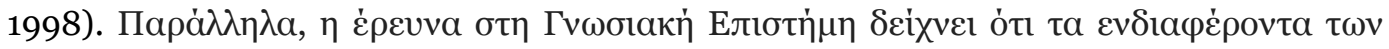

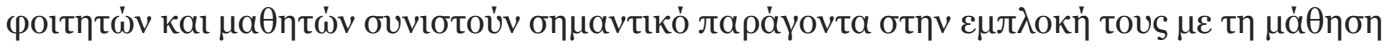

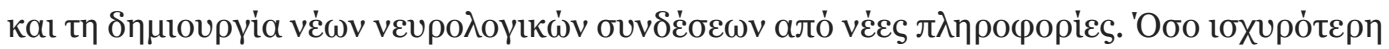

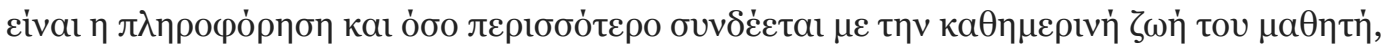

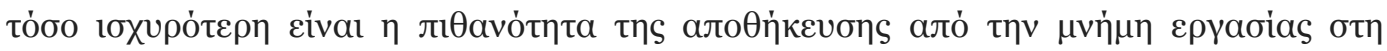

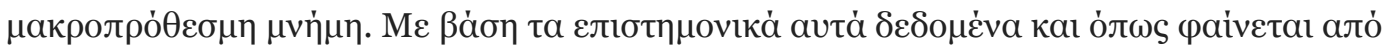

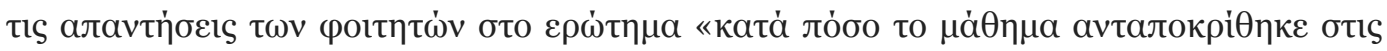

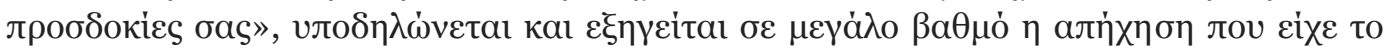

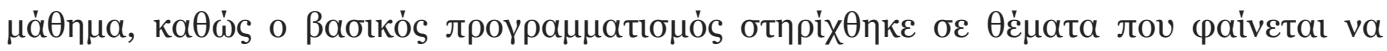

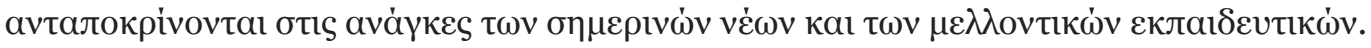

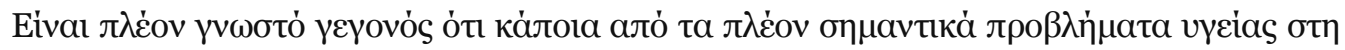

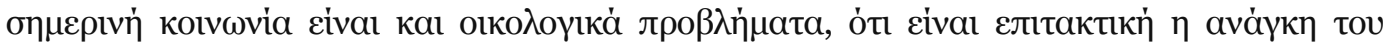

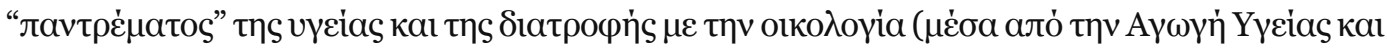

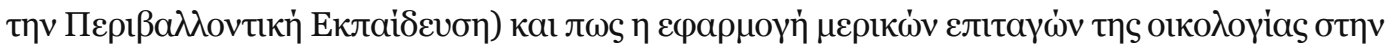

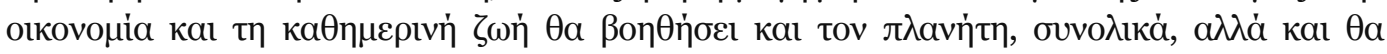

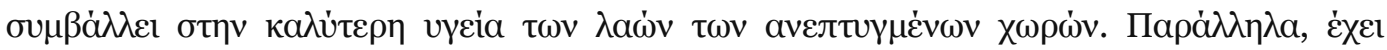

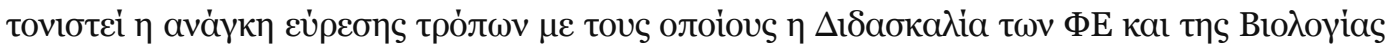

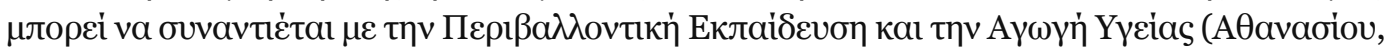

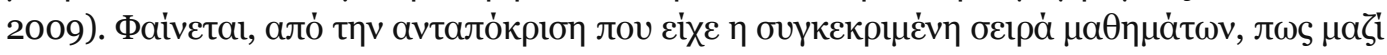




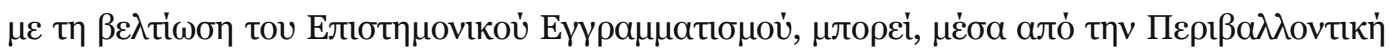

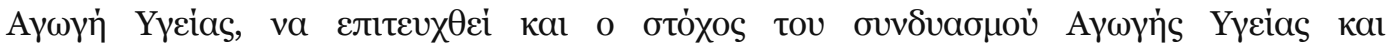

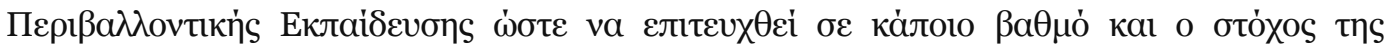

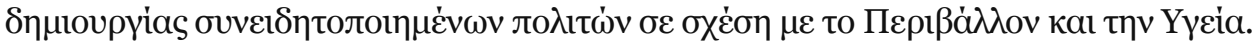

\section{Bıßлıоррачі́a}

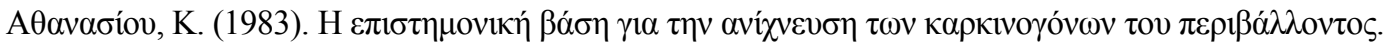

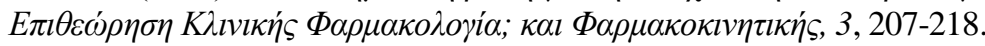

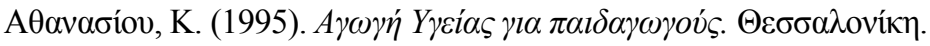

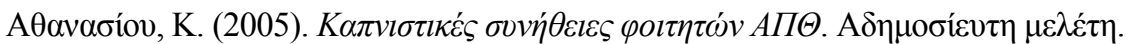

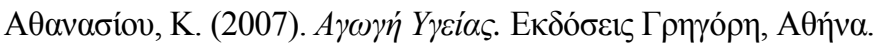

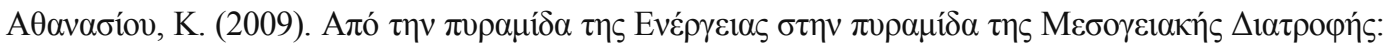

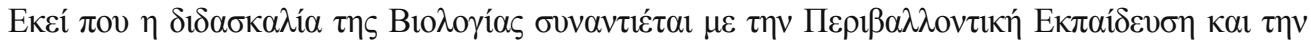

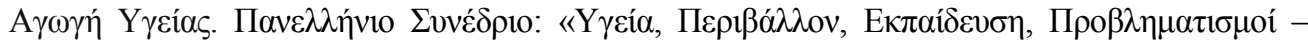

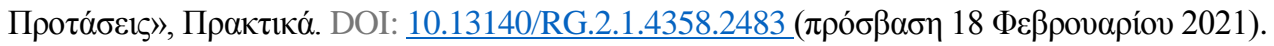

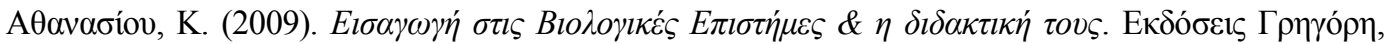
A $\theta \eta \dot{v} \alpha$.

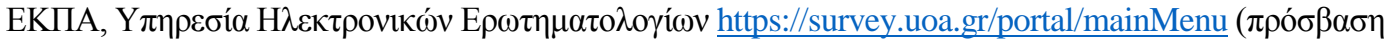
18 Фєßроvарíov 2021).

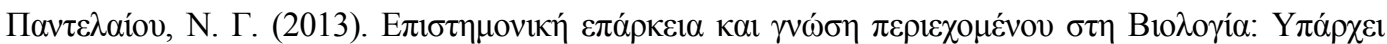

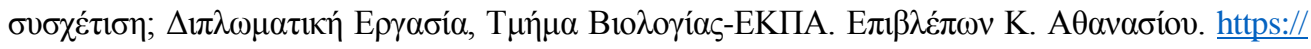

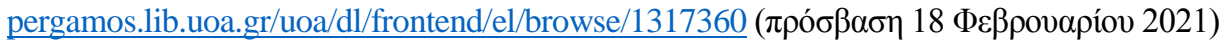

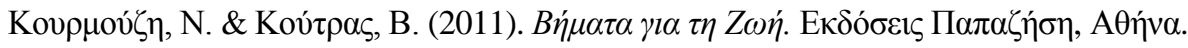

American Cancer Society (2008). Cancer facts and figures.

Ames B.N. (1983). Dietary carcinogens and anticarcinogens. Science, 221, 1256-1264.

Athanasiou, K. \& Kyrtopoulos, S.A (1983). Mutagenic and clastogenic effects of organic extracts from the Athenian drinking water. The Science of the Total Environment, 27, 113-120.

Athanasiou, K., I. Arzimanoglou, C. Piccoli \& Yamasaki, H. (1987). Mutagenicity, clastogenicity and in-vitro transforming ability of particulates from Athens air. Cell Biology and Toxicology, 3, 301-307.

Athanasiou, K., \& Papadopoulou, P. (2011). Conceptual Ecology of the Evolution acceptance among Greek education students: knowledge, religious practices and social influences. International Journal of Science Education, 34, 903-924.

Athanasiou, K., Viras, L.G \& Siskos, P.A. (1986). Mutagenicity and polycyclic aromatic hydrocarbons analysis of ambient airborne particles collected in Athens, Greece. The Science of the Total Environment, 52, 201-209. 
Athanasiou, K. \& Macris, G. (1995). Smoking and Cancer: Exploring the level of knowledge and attitudes of Greek youngsters and parents. In K. Slama (Eds.), Tobacco and Health (pp. 623-625). Plenum Press, New York and London.

Currie, C., Zanotti, C., Morgan, A., Currie, D. de Looze, M., Roberts, C., Samdal, O., Smith, O. \& Barnekow, V. (2012). Social determinants of health and well-being among young people. Health Behavior in School-aged Children (HBSC) study: international report from the 2009/2010 survey. Copenhagen, WHO Regional Office for Europ (Health Policy for Children and Adolescents, No. 6).

Charalabopoulos, K., Makris, G., Charalabopoulos, A., Golias, C. \& Athanasiou, K. (2011). Public knowledge, beliefs and practices in Greece about cancer etiology and prevention. East Mediterranean. Health Journal, 17(5), 392-397.

Doll, R. (1998). Epidemiological evidence of the effects of behavior and the environment on the risk of human cancer. Recent Results in Cancer Research, 154, 3-21.

Gough, A., Marshall, A., Matthews, R., Milne, G., Tytler, R. \& White, G. (1998) Science Baseline Survey. Deakin University Faculty of Education Consultancy and Development Unit for the Department of Education, Victoria, September 1998. Confidential Consultancy Report.

Heddle, J.A. andAthanasiou, K. (1975). Mutation rates, genome size and their relation to the "rec" concept. Nature, 258, 359-361.

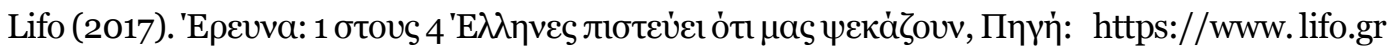

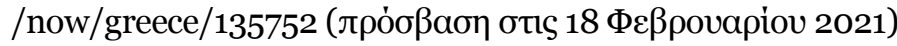

Makris, G., Charalampopoulos, K.A \& Athanasiou, K. (1994). Estimating the level of knowledge of Greek students on cancer etiology and ways of prevention. European Journal of Cancer prevention, 3, 443-450.

McCann, I., Choi, Yamasaki, E. \& Ames, B.N. (1975). Detection of carcinogens as mutagens in the Salmonella/microsome test: assay of 300 chemicals. Proceedings of the National Academies of Sciencecs of the United States of America (PNAS), 72(12), 5135-5139.

McComas, W. F. (2008). Seeking historical examples to illustrate key aspects of the nature of science. Science \& Education, 17(2), 249-263.

Peto, R. (1985). In IARC Monographs on the Evaluation of the Carcinogenic Risk of Chemicals to Humans, Vol. 38, Lyon.

Science in Schools Research Project Manual (2001). Burwood: Deakin University.

von Glasersfeld, E. (1998). Cognition, Construction of Knowledge, and Teaching. In Matthews, M.R (eds.) Constructivism in Science Education (pp.11-30). Dordrecht: Springer. 


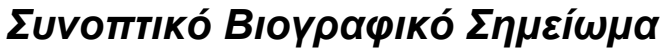

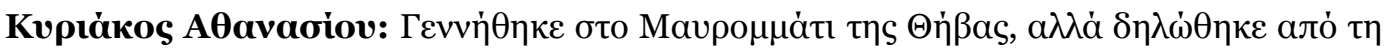

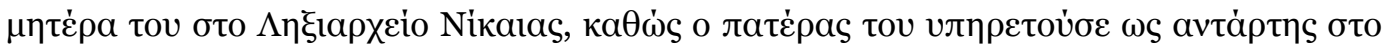

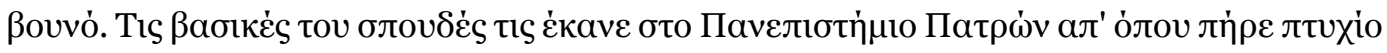

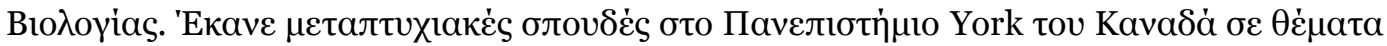

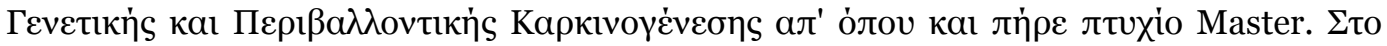

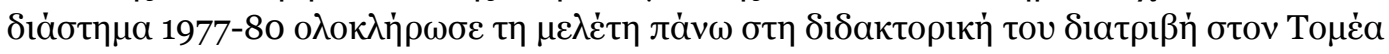

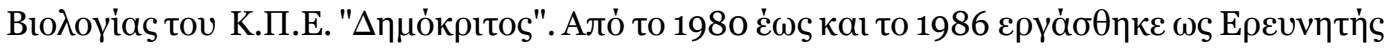

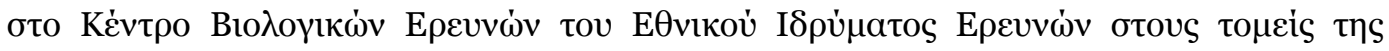

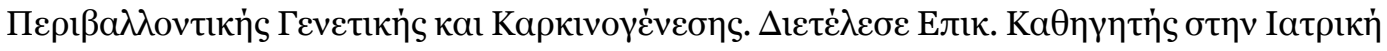

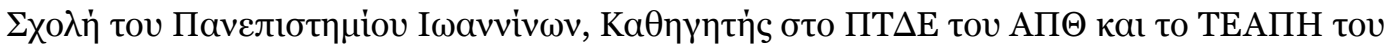

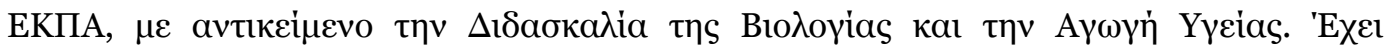

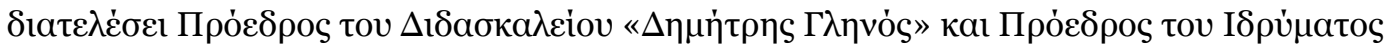

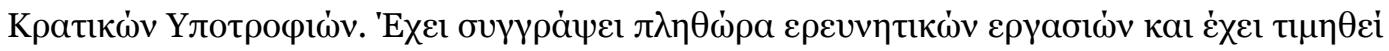

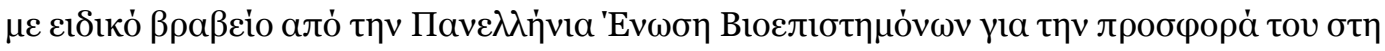

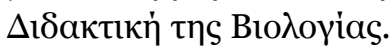

